\title{
Pre-print: Mid-water Current Aided Localization for Autonomous Underwater Vehicles
}

\author{
Lashika Medagoda - Stefan B. Williams - Oscar Pizarro - James C. \\ Kinsey · Michael V.Jakuba
}

Received: date / Accepted: date

\begin{abstract}
Survey-class Autonomous Underwater Vehicles (AUVs) typically rely on Doppler Velocity Logs (DVL) for precision localization near the seafloor. In cases where the seafloor depth is greater than the DVL bottom-lock range, localizing between the surface and the seafloor presents a localization problem since both GPS and DVL observations are unavailable in the midwater column. This work proposes a solution to this problem that exploits the fact that current profile layers of the water column are near constant over short time scales (in the scale of minutes). Using observations of these currents obtained with the Acoustic Doppler Current Profiler (ADCP) mode of the DVL during descent, along with data from other sensors, the method discussed herein constrains position error. The method is validated using field data from the Sirius AUV coupled with view-based Simultaneous Localization and Mapping (SLAM) and on descents up to $3 \mathrm{~km}$ deep with the Sentry AUV.
\end{abstract}

Keywords AUV - ADCP · Underwater - Localization · Mid-water $\cdot$ Navigation

Lashika Medagoda

Robotics Innovation Center, German Research Center for Artificial Intelligence, DFKI Bremen, Robert-Hooke-Strasse 1, 28359 Bremen, Germany

lashika.medagoda@dfki.de

Stefan B. Williams · Oscar Pizarro

Australian Centre for Field Robotics, University of Sydney, Sydney NSW 2006 Australia

\{stefanw, o.pizarro\}@acfr.usyd.edu.au

James C. Kinsey · Michael V. Jakuba

Deep Submergence Laboratory, Woods Hole Oceanographic Institution, Applied Ocean Physics \& Engineering, 266 Woods Hole Rd. MS 07, Woods Hole, MA 02543-1050, USA \{jkinsey, mjakuba\}@whoi.edu

\section{Introduction}

Autonomous Underwater Vehicles (AUVs) have emerged as the platform of choice for a wide variety of survey tasks in the commercial, oceanographic, and military domains. Within the oceanographic community, AUVs are used for tasks such as seafloor mapping (e.g., Caress et al (2012); Kelley et al (2005)), habitat monitoring (e.g., Williams et al (2012)), optical surveys (e.g., Singh et al (2004a)), high-resolution magnetic surveys (Tivey et al (1998)), climate change research (Schofield et al (2010)) and localizing hydrothermal and hydrocarbon plumes (German et al (2008) and Camilli et al (2010), respectively). An advantage of AUVs over other ocean observation methods is the potential for reduced costs (i.e., decreased dependence on manned surface vessels) as well as increased mission duration - especially as long-range AUVs mature (Hobson et al (2012); Furlong et al (2012)) and longer duration missions (on the order of weeks or months) increasingly become a reality.

As with present AUV operations, navigation is a crucial element for long duration missions. This problem is especially acute in deep water where the time and power costs associated with surfacing for Global Positioning System (GPS) position fixes is prohibitively high. A variety of methods exist in deep water (Kinsey et al (2006); Paull et al (2014) provide surveys of the state of the art) with Doppler Velocity Log (DVL) navigation (e.g., Brokloff (1994); Kinsey and Whitcomb (2004)) being the predominant method for AUVs operating within 200-300m of the seafloor. Precision navigation in the mid-water column (i.e., below the sea surface and more than a few hundred meters from the seafloor) is more difficult (Kinsey et al $(2006,2014)$ ) and presents challenges for AUVs operating in this region. This implies that few methods are available for 
deep-diving AUVs during descent from the sea surface to the ocean floor. For example, the Sentry AUV conducts operations at depths up to $6000 \mathrm{~m}$ and during long descents can horizontally drift hundreds of meters from the launch position. Like most deep-diving AUVs, Sentry relies on Ultra Short Base Line (USBL) navigation (e.g., Peyronnet et al (1998)) during these descents and the position of the AUV is tracked from the ship and, upon acquisition of DVL bottom-lock near the seafloor, the AUV's USBL position is transmitted via acoustic modem to the AUV thereby enabling the necessary navigation correction. This paradigm has two drawbacks: (1) USBL navigation is noisy and provides position estimates at $\sim 10$ s intervals; and (2) requires the continuous presence of an expensive dedicated surface vessel. Long Base Line (LBL) can also provide navigation during descent but require time-consuming deployment of transponders (Hunt et al (1974); Yoerger et al (2007)). Thus, developing methods that improve navigation in the mid-water column while reducing the dependency on surface vessels or a-priori infrastructure would reduce the costs and supervision associated with AUV missions.

This paper proposes a localization solution in the mid-water column that takes advantage of the near constant current profile layer velocities over short time periods. A common assumption in the oceanographic community is that the water currents are constant during the period in which they are observed, a period of minutes (Visbeck (2002)). For deep diving AUVs operating in the open ocean, such as Sentry, currents are constant over time scales of hours. The AUVs typically descend at $30-40 \mathrm{~m} / \mathrm{min}$ and ADCPs possess ranges of $40-120 \mathrm{~m}$. Thus the observable period for each water current velocity layer is on the order of minutes during which the ocean currents will change negligibly. When fused with other sensor observations, we show that there is improved localization performance for AUVs operating in both shallow and deep water.

This paper is organized as follows: Section 2 provides a literature review with a focus on mid-water column localization. Section 3 presents our ADCP-aided localization method. Section 4 applies the ADCP localization method to 2D and 3D simulated cases. Section 5 implements localization with the ADCP sensoraiding using real data on a shallow water instance coupled with view-based SLAM. Section 6 applies the filter to data collected during deep water missions. Section 7 compares the experimental results presented in this paper and discusses their implications. Section 8 concludes the paper with a summary of the contributions along with suggestions for the direction of future work.
Previous work by the authors has reported the theory of ADCP-aided localization, illustrative examples and initial experimental results (Medagoda et al (2010, 2011)) — these prior results are summarized here for completeness (Sections 3, 4.1 and 5 respectively). This paper expands on this prior work in a number of ways. First, this paper provides a more extensive literature review including how this work distinguishes itself from other work in ADCP-aided navigation (Section 2). Second, we introduce a strategy to marginalize out older states for computational efficiency and study the implication of adding carrier phase GPS (Section 4.2). The effects of these improvements are studied using new simulations for deeper water and longer timescales (Section 4.2.2). Third, we implement and assess this method on data obtained on deep-water AUV dives and report the details associated with implementing this method in deep-water including incorporating sensor error checking information (Section 6). Finally, this paper summarizes and compares all of the simulations and experiments, and analyzes the drivers for localization performance using this method (Section 7).

\section{Mid-Water Localization}

AUV georeferencing is typically achieved by fusing information from multiple sensors to estimate the vehicle's position and orientation in space. Depth and attitude estimates can be directly obtained from existing sensors - e.g., hydrostatic pressure measurements provide accurate absolute depth information and Attitude Heading Reference Systems (AHRSs) provide orientation measurements (though there is significant variance in accuracy, cost, and power (Kinsey et al (2006))). Horizontal XY position estimates are more difficult to obtain. GPS is available on the ocean surface, but does not penetrate the water. DVLs provide velocity-overground information when within bottom-lock distance of the seafloor and when coupled with an attitude sensor provide dead reckoning. A variety of frameworks are employed to fuse navigation sensor data; such as the Extended Kalman Filter (EKF) framework, although other probabilistic estimation techniques can be utilized (Paull et al (2014)). This allows for near-optimal estimation, although optimality can be traded off for stability in alternative implementations (Kinsey et al (2014)). GPS and DVL observations are unavailable in the mid-water column and thus other solutions are required for localization. 


\subsection{Time-of-Flight Acoustic Localization Methods}

Traditional solutions to georeference during a descent or ascent include time-of-flight acoustic localization methods such as USBL and LBL. USBL requires a ship to track the vehicle acoustically, thus requiring a tending vessel for the duration of the mission. This may not always be possible, for example under ice. LBL requires an acoustic transponder network, which includes deploying and surveying in the beacons. To reduce the set-up requirements, single fixed beacons can be utilized (Paull et al (2014)). Regardless of the method, the mission will be limited to the range of the acoustic beacon network — typically $1-10 \mathrm{~km}$ ranges with $\sim 10$ m accuracy (Kinsey et al (2006); McPhail and Pebody (2009)).

\subsection{Localization using an IMU}

IMUs employ accelerometers and gyroscopes to provide body-relative accelerations and rotation rates to constrain the position, velocity and attitude estimates through integration of the outputs. Given an IMU capable of gyrocompassing (observing the locally projected $15 \mathrm{deg} / \mathrm{hr}$ rotation of the Earth), the position error growth is approximately $\delta p \approx \delta \omega \mathbf{R}_{0} t$ (Titterton and Weston (2004)). $\delta p$ is the position drift, $\delta \omega$ is the gyro bias, $\mathbf{R}_{0}$ is the radius of the Earth, and $t$ is time.

Thus a navigation-grade IMU (often in excess of $\$ 100 \mathrm{k}$ USD (Kinsey et al (2006)) with $0.01^{\circ} / \mathrm{hr}$ gyro bias, will achieve $\sim 1 \mathrm{~km} / \mathrm{hr}$ position drift without aiding. Alternatively, a tactical grade IMU (approximately $\$ 16 \mathrm{k}$ USD) with $1^{\circ} / \mathrm{hr}$ gyro bias will achieve $\sim 100$ $\mathrm{km} / \mathrm{hr}$ position drift without aiding. Once underwater, when the vehicle is within DVL range of the seafloor, $\sim 0.2 \%$ distance traveled position error growth $(2 \sigma)$ is possible when DVL is coupled with a navigationgrade IMU (Napolitano (2004); iXSea (Accessed 22-032012)).

\subsection{Localization using a DVL}

The DVL operates by sending out an acoustic pulse and measures the Doppler shift of the return pulse from the seafloor (colloquially referred to as 'DVL bottom-lock'). By using four sensor beams with different orientations, the $3 \mathrm{D}$ velocity of the DVL can be determined. The fourth sensor provides redundancy in the estimation of the current profile velocities (Gordon (1996)). The result is a velocity estimate with an accuracy of $\sim 10$ $\mathrm{mm} / \mathrm{s}(2 \sigma)$ for a $1200 \mathrm{kHz}$ DVL.
Low-frequency $(300 \mathrm{kHz})$ DVL can be in continuous use for altitudes less than $\sim 200 \mathrm{~m}$. The DVL sensor provides measurements of the seafloor-relative velocity of the AUV. By combining this information with an appropriate heading reference, the observations can be placed in a global reference frame and integrated for underwater dead reckoning (Brokloff (1994); Whitcomb et al (1999); Kinsey and Whitcomb (2004)).

\subsubsection{Localization using the DVL Water-Track Mode}

The DVL water-track mode provides a measurement of the velocity of the AUV relative to a user-programmable water sampling volume, instead of the seafloor like the DVL. It is a built-in capability of DVLs requiring only software configuration changes (Gordon (1996)). It operates by sending out an acoustic pulse and relying on scatterers, such as plankton, to reflect back the pulse. Using the Doppler effect, the velocity of the scatterers relative to the instrument can be determined. Since it is assumed that the scatterers move with the water currents, the DVL water-track measures the velocity of the water column currents relative to the sensor which can be used for dead-reckoning — albeit not referenced to the seafloor (Brokloff (1997)). By time-gating the signal for a specific time period, a user-programmable water sampling volume is measured. This method assumes that currents are horizontally homogeneous across a water layer to arrive at a water current estimate (Gordon (1996)).

\subsubsection{Acoustic Doppler Current Profiler (ADCP)}

The ADCP (Brumley et al (1991)) is another mode of the same DVL sensor and operates similarly to the DVL water-track mode. The ADCP processes returns at different times instead of one. This allows the sensor to measure water currents at different ranges, segmenting the observation into measurement cells. This is illustrated in Figure 1.

By using 4 differently aligned sensor beams and assuming horizontally homogeneous currents, the $3 \mathrm{D}$ velocity of the current can be determined in a similar manner to the DVL mode. The result is a current estimate with an accuracy of $\sim 20 \mathrm{~mm} / \mathrm{s}(2 \sigma)$ observing 2 $\mathrm{m} / \mathrm{s}$ currents for a $1200 \mathrm{kHz}$ instrument ${ }^{1}$.

Acoustic Doppler Current Profilers (ADCPs) have traditionally been used by the oceanographic community to estimate water current profiles for scientific studies. The existing method applies least-squares to fuse lowered ADCP and DVL bottom-lock information (Visbeck (2002)). This approach does not address ADCP

$109 / \mathbf{1 9} / 2009$ email from Teledyne RD Instruments 


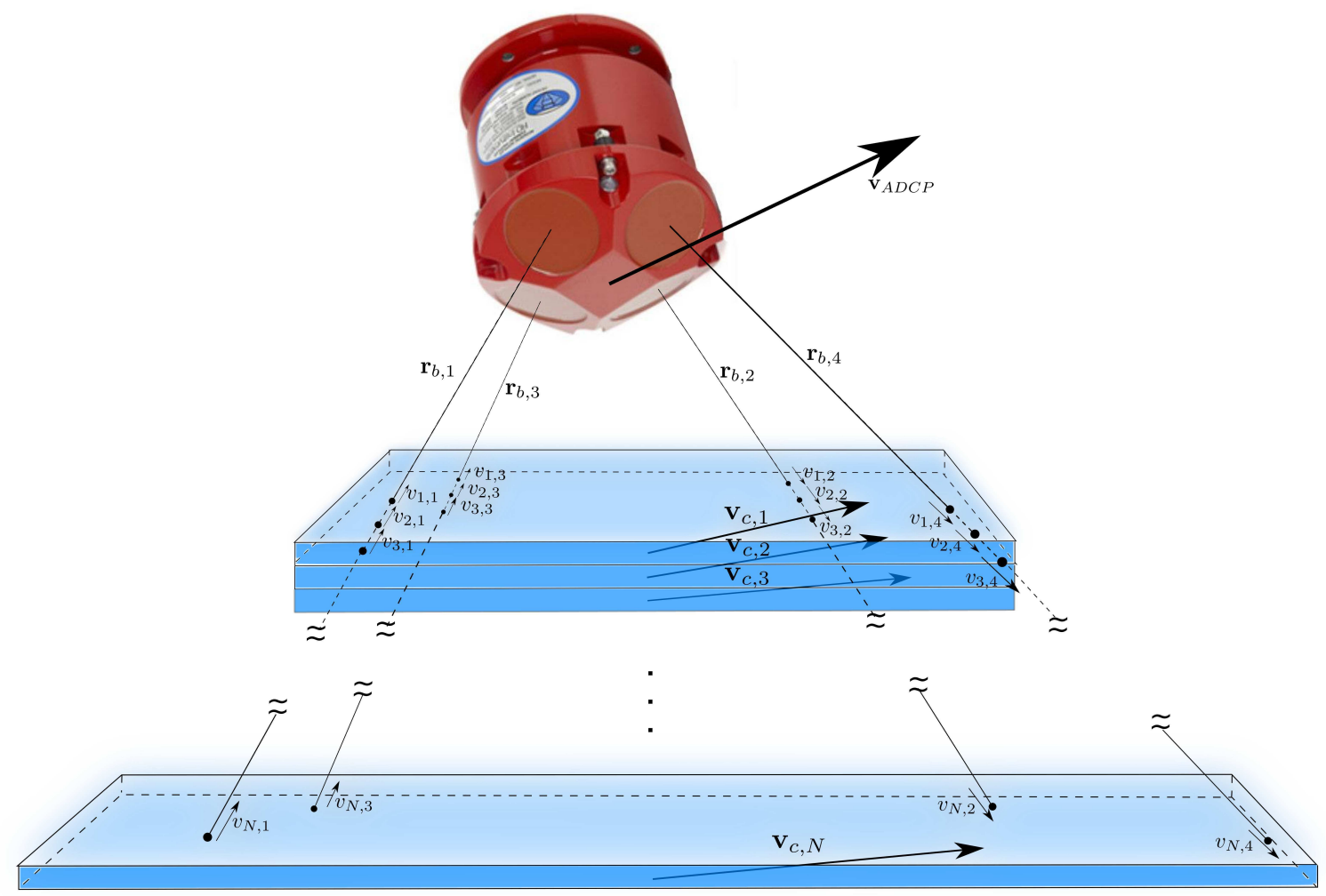

Fig. 1 - The ADCP mode of the DVL sensor operates by looking at the returns from scatterers in the water column along each of the 4 beams. This information can be combined to arrive at the $3 \mathrm{D}$ velocity of the water currents relative to the ADCP sensor in a similar way to the DVL sensor. By time-gating the signal return, differently distanced water currents can be estimated. This is in contrast to the DVL water-track mode, since the ADCP mode measures multiple water currents spread spatially.

sensor biases (Gordon (1996)) and sensor uncertainties as their effects on the overall current profile are assumed minimal. However, biases have implications on the velocity estimates of an AUV-mounted ADCP used for localization during descent or ascent. Furthermore, this prior work does not seek to estimate the ADCP position.

\subsection{Vehicle Model Based Navigation}

Using a model of the vehicle dynamics to predict how the vehicle will move given the estimated control actions (such as thrusters) allows additional information to be fed into the localization algorithms. In Hegrenaes and Hallingstad (2011), the vehicle model aids the localization by modeling the AUV dynamics given control actions and a local water current estimate. This implementation uses an IMU as the prediction stage of an Extended Kalman Filter (EKF). The vehicle model provides input to the update stage, facilitated by adding a correlation term to the state vector, which has a firstorder Markov bias model. A navigation-grade IMU cou- pled with a vehicle model and DVL water-track mode estimates of currents can achieve $\sim 60 \mathrm{~m}$ over 30 minutes $(2 \sigma)$ position uncertainty growth after acquiring DVL bottom-lock (Hegrenaes and Berglund (2009)). The assumption is a time-varying current in the measured water sampling volume. Prior to DVL bottom-lock, the position uncertainty growth is $\sim 450 \mathrm{~m}$ over 30 minutes (Hegrenaes and Berglund (2009)). As time progresses, this conservative constraint approaches the worst case water current velocity uncertainty.

\section{ADCP Sensor Aiding with Water Layers}

An alternative to using the DVL water-track mode is to use the ADCP mode to provide spatially-mapped finerdepth resolution current estimation. This introduces the possibility of improved vehicle motion estimates. The standard parametrization of the ocean for lowered $\mathrm{ADCP}$ is to layer the ocean into discrete, isocurrent layers (Visbeck (2002)), or depth cells. This standard will be applied in this paper. This relies on the assump- 
tion of horizontal homogeneity across the water current layer (Gordon (1996)).

In parallel to our work (Medagoda et al (2010, 2011)) was that of Stanway (2011, 2012). In Stanway's work, a least squares approach to estimate water currents and vehicle pose was applied. GPS, ADCP, DVL and magnetic compass heading are utilized for localization. His work addresses practical ADCP implementation issues for deep water localization, such as ADCP sensor configuration and diagnostics during AUV ascents, which complements our work. The recursive least squares approach is similar to the computational complexity of the delayed-state filter (with marginalization) used in this paper.

In comparison, this work has explored the incorporation of SLAM and retaining the entire state history of the filter for relinearization purposes. Reinearization (Kaess et al (2011)) of the Jacobians is undertaken to improve consistency in linearizing filters such as nonlinear least squares and the EKF. This is due to the linearization point being around the estimate (which contains error) and not the unknown true state. Through smoothing, past states are better estimated. The Jacobians then can be recalculated. Relinearization requires increased computational cost. Due to a more general framework in this paper, the IMU, TDCP GPS and view-based SLAM can be incorporated. The incorporation of ADCP bias estimation in the filter is undertaken in this paper. Stanway's work does not incorporate bias estimation, nor are uncertainty bounds analyzed. The illustrative example in Section 4.1 outlines how both Stanway's and this paper's method works. An uncertainty analysis of our method is also undertaken.

\subsection{ADCP estimation and navigation aiding process}

We assume that initially the AUV has position and velocity estimates in the navigation frame at the sea surface from GPS, illustrated in Figure 2(a). With the initial measurement from the ADCP sensor, body-relative water depth cell velocities below the vehicle are observed using each ADCP measurement cell. These observations can be used to estimate the full current profiles in the navigation frame by using the estimated vehicle velocity at the surface.

The vehicle then submerges and GPS measurements stop. When another ADCP measurement is made, the vehicle re-observes the same depth cells, shown in Figure 2(b). Given the estimated water current velocity of the re-observed depth cell and the body-relative velocity of these depth cells from the ADCP, a filter can simultaneously update the estimate of the vehicle velocity and current profile velocities as shown in Figure 2(c). This assumes that the water current velocity in this depth cell remains constant, which is realistic over a re-observation period of minutes (Visbeck (2002)). This is in addition to the horizontal homogeneity assumption for the ADCP footprint during the descent. New water current velocity states are initialized when ascending due to temporal and spatial changes.

New depth cells can also now be estimated as the vehicle changes depth as shown in the bottom right of Figure 2(c) in red. The result is an estimate of the vehicle motion and a water column current profile. When the vehicle is within DVL range of the seafloor, this velocity constraint on the vehicle is also incorporated into the filter.

\subsection{Extended information filter with current profiling}

Vehicle pose states, ADCP bias (outlined in Section 3.2.2) states and water current velocity are all estimated simultaneously. Water velocity states are parameterized as isocurrent depth cells, each with an associated velocity vector. An Extended Information Filter (EIF) is applied to estimate the states of the vehicle given the various vehicle sensor measurements (Walter et al (2007)). This allows the water current layer depth cell states to be estimated, along with maintaining the correlations between the states. The EIF also allows relinearization (Kaess et al (2011)) of the Jacobians if required and can incorporate view-based SLAM (Mahon et al (2008)) if applicable, which is not computationally feasible with a naive EKF implementation. It also enables the entire state history of the vehicle to be viewed for analysis, as it acts as a delayed state smoother.

Vehicle pose states such as position, velocity and attitude, ADCP bias states and water current velocity states are stored in a state vector of the form

$$
\hat{\mathbf{x}}^{+}\left(t_{k}\right)=\left[\begin{array}{c}
\hat{\mathbf{x}}_{P_{1}}^{+}\left(t_{k}\right) \\
\vdots \\
\hat{\mathbf{x}}_{P_{n_{P}}}^{+}\left(t_{k}\right) \\
\hat{\mathbf{x}}_{b_{c, 1}}^{+}\left(t_{k}\right) \\
\vdots \\
\hat{\mathbf{x}}_{b_{c, n_{b}}}^{+}\left(t_{k}\right) \\
\hat{\mathbf{x}}_{v_{c, n}}^{+}\left(t_{k}\right) \\
\vdots \\
\hat{\mathbf{x}}_{v_{c, n_{v}}^{+}}^{+}\left(t_{k}\right)
\end{array}\right]=\left[\begin{array}{c}
\hat{\mathbf{x}}_{P}^{+}\left(t_{k}\right) \\
\hat{\mathbf{x}}_{b_{c}}^{+}\left(t_{k}\right) \\
\hat{\mathbf{x}}_{v_{c}}^{+}\left(t_{k}\right)
\end{array}\right]
$$

where $\hat{\mathbf{x}}_{P}^{+}\left(t_{k}\right)=\left[\hat{\mathbf{x}}_{P_{1}}^{+\mathrm{T}}\left(t_{k}\right), \ldots, \hat{\mathbf{x}}_{P_{n_{P}}}^{+\mathrm{T}}\left(t_{k}\right)\right]^{\top}$ is a vector of past and present pose states where $n_{P}$ is the number of vehicle pose states, $\hat{\mathbf{x}}_{b_{c}}^{+}\left(t_{k}\right)=\left[\hat{\mathbf{x}}_{b_{c, 1}}^{+\mathrm{\top}}\left(t_{k}\right), \ldots, \hat{\mathbf{x}}_{b_{c, n_{b}}}^{+\mathrm{T}}\left(t_{k}\right)\right]^{\top}$ 


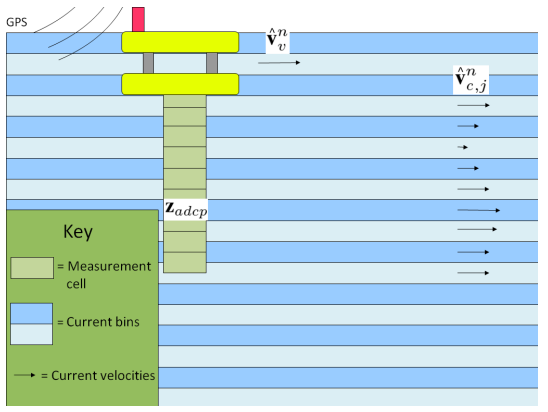

(a)

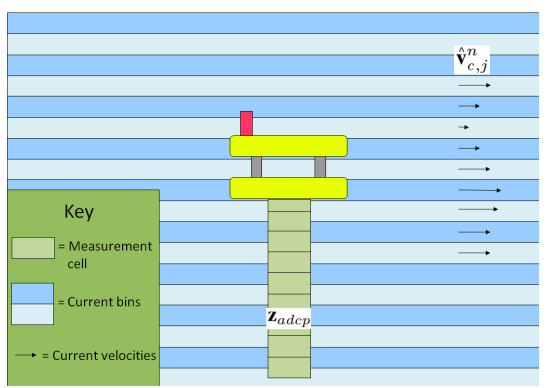

(b)

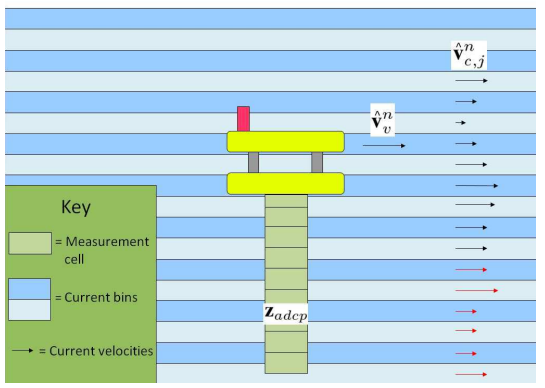

(c)

Fig. 2 - ADCP-aiding method sequence (a) Initial GPS position and velocity are known, and water velocities can be estimated. (b) The AUV moves, and re-observes the same depth cells. (c) The estimated AUV velocity in the world frame can be updated, and new depth cells, shown in red (bottom right), can be added to the filter.

is a vector of past and present $\mathrm{ADCP}$ bias states where $n_{b}$ is the number of ADCP bias states and $\hat{\mathbf{x}}_{v_{c}}^{+}\left(t_{k}\right)=$ $\left[\hat{\mathbf{x}}_{v_{c, 1}}^{+\mathrm{\top}}\left(t_{k}\right), \ldots, \hat{\mathbf{x}}_{v_{c, n_{v}}}^{+\mathrm{\top}}\left(t_{k}\right)\right]^{\top}$ is a vector of past and present ADCP water current velocity states where $n_{v}$ is the number of water current velocity states. In this this paper, $n_{P}=9 . n_{b}$ is initialized at 60 in this paper, with the state number increasing at a desired rate (in this case every 5 minutes). $n_{v}$ begins at 60 , and then increases as more water current states are observed during vertical motion. As shown later, these states can be marginalized if no longer observed, without effecting the estimation of present states. This prevents an unbounded increase in the computation cost. The covariance between the pose states and the water current states are in the form

$\hat{\mathbf{P}}^{+}\left(t_{k}\right)=\left[\begin{array}{lll}\hat{\mathbf{P}}_{P P}^{+}\left(t_{k}\right) & \hat{\mathbf{P}}_{P b_{c}}^{+}\left(t_{k}\right) & \hat{\mathbf{P}}_{P v_{c}}^{+}\left(t_{k}\right) \\ \hat{\mathbf{P}}_{P b_{c}}^{+\top}\left(t_{k}\right) & \hat{\mathbf{P}}_{b_{c} b_{c}}^{+}\left(t_{k}\right) & \hat{\mathbf{P}}_{b_{c} v_{c}}^{+}\left(t_{k}\right) \\ \hat{\mathbf{P}}_{P v_{c}}^{+T}\left(t_{k}\right) & \hat{\mathbf{P}}_{b_{c} b_{c}}^{+T^{+}}\left(t_{k}\right) & \hat{\mathbf{P}}_{v_{c} v_{c}}^{+}\left(t_{k}\right)\end{array}\right]$

In the information form, the filter maintains the matrix $\mathbf{Y}$, which is the inverse of the covariance matrix

$\hat{\mathbf{Y}}^{+}\left(t_{k}\right)=\left[\hat{\mathbf{P}}^{+}\left(t_{k}\right)\right]^{-1}$

and the information vector $\mathbf{y}$, which is related to the state estimate by

$\hat{\mathbf{y}}^{+}\left(t_{k}\right)=\hat{\mathbf{Y}}^{+}\left(t_{k}\right) \hat{\mathbf{x}}^{+}\left(t_{k}\right)$

The information vector has the form

$\hat{\mathbf{y}}^{+}\left(t_{k}\right)=\left[\begin{array}{l}\hat{\mathbf{y}}_{P}^{+}\left(t_{k}\right) \\ \hat{\mathbf{y}}_{b_{c}}^{+}\left(t_{k}\right) \\ \hat{\mathbf{y}}_{v_{c}}^{+}\left(t_{k}\right)\end{array}\right]$

and the information matrix has the form

$$
\hat{\mathbf{Y}}^{+}\left(t_{k}\right)=\left[\begin{array}{ccc}
\hat{\mathbf{Y}}_{P P}^{+}\left(t_{k}\right) & \hat{\mathbf{Y}}_{P b_{c}}^{+}\left(t_{k}\right) & \hat{\mathbf{Y}}_{P v_{c}}^{+}\left(t_{k}\right) \\
\hat{\mathbf{Y}}_{P b_{c}}^{+P}\left(t_{k}\right) & \hat{\mathbf{Y}}_{b_{c} b_{c}}^{+}\left(t_{k}\right) & \hat{\mathbf{Y}}_{b_{c} v_{c}}^{+}\left(t_{k}\right) \\
\hat{\mathbf{Y}}_{P v_{c}}^{+\top}\left(t_{k}\right) & \hat{\mathbf{Y}}_{b_{c} b_{c}}^{++}\left(t_{k}\right) & \hat{\mathbf{Y}}_{v_{c} v_{c}}^{+}\left(t_{k}\right)
\end{array}\right]
$$

Observations, which include ADCP measurements, are assumed to be made according to

$\mathbf{z}\left(t_{k}\right)=\mathbf{h}\left(\mathbf{x}\left(t_{k}\right)\right)+\nu\left(t_{k}\right)$

in which $\mathbf{z}\left(t_{k}\right)$ is an observation vector, $\mathbf{h}\left(\mathbf{x}\left(t_{k}\right)\right)$ is the sensor model relating states to observations, and $\nu\left(t_{k}\right)$ is a vector of observation noise with covariance $\mathbf{R}\left(t_{k}\right)$. New information from sensor measurements are incorporated into the information vector and matrix

$\hat{\mathbf{y}}^{+}\left(t_{k}\right)=\hat{\mathbf{y}}^{-}\left(t_{k}\right)+\mathbf{i}\left(t_{k}\right)$

$\hat{\mathbf{Y}}^{+}\left(t_{k}\right)=\hat{\mathbf{Y}}^{-}\left(t_{k}\right)+\mathbf{I}\left(t_{k}\right)$

in which

$$
\begin{aligned}
\mathbf{i}\left(t_{k}\right)= & \nabla_{x}^{\top} \mathbf{h}\left(t_{k}\right) \mathbf{R}^{-1}\left(t_{k}\right)\left(\mathbf{z}\left(t_{k}\right) \ldots\right. \\
& \left.-\mathbf{h}\left(\hat{\mathbf{x}}^{-}\left(t_{k}\right)\right)+\nabla_{x} \mathbf{h}\left(t_{k}\right) \hat{\mathbf{x}}^{-}\left(t_{k}\right)\right) \\
\mathbf{I}\left(t_{k}\right)= & \nabla_{x}^{\top} \mathbf{h}\left(t_{k}\right) \mathbf{R}^{-1}\left(t_{k}\right) \nabla_{x} \mathbf{h}\left(t_{k}\right)
\end{aligned}
$$

where $\hat{\mathbf{x}}^{-}\left(t_{k}\right)$ is the a priori state estimate and $\nabla_{x} \mathbf{h}\left(t_{k}\right)$ is the Jacobian of the observation with respect to the state. Using this framework, the recursive non-linear weighted least squares solution to the states can be estimated.

\subsubsection{ADCP observation equation}

Given the 3D velocities output from the ADCP, the observation function for each ADCP measurement is

$\mathbf{h}_{A D C P, i}=\mathbf{C}_{n}^{b}\left(-\mathbf{v}_{v}^{n}+\sum \mathbf{W}_{j} \mathbf{v}_{c, j}^{n}\right)+\mathbf{b}_{c, i}+\nu_{A D C P}$

where:

- $\mathbf{h}_{A D C P, i}=$ ADCP measured current vector in the $\mathrm{i}^{\text {th }}$ measurement cell

- $\mathbf{C}_{n}^{b}=$ Coordinate transform from navigation/world frame to $\mathrm{ADCP} /$ body frame

- $\mathbf{v}_{v}^{n}=$ Vehicle velocity in the world/navigation frame

- $\mathbf{W}_{j}=$ Weighting function for each water current velocity from depth cell $\mathrm{j}$, outlined in Gordon (1996) 
$-\mathbf{v}_{c, j}^{n}=$ water current velocity from depth cell $\mathrm{j}$. Each depth cell contains a current velocity state in the $\mathrm{X}$ and $\mathrm{Y}$ direction, which represents the average velocity of the current through that layer

$-\mathbf{b}_{c, i}=$ Bias in the $\mathrm{i}^{t h}$ measurement cell in the body frame

$-\nu_{A D C P}=$ Random noise in the ADCP measurement, with standard deviation given by the sensor manufacturer

The ADCP measurement equation correlates the vehicle velocity and water current velocities.

\subsubsection{ADCP biases}

It is important to consider any biases in the measurements, as they will provide a large source of error, and will result in the filter becoming overconfident if left unmodeled. Sources of ADCP biases include measurement cell dependent biases, such as (a) beam and sensor misalignment, $(b)$ beam geometry; and (c) signal/noise ratio and biases dependent on changing depth, including temperature, pressure, scatterers and sound speed estimate error (Gordon (1996); Atkinson (2008)). In this paper, these ADCP biases are estimated as the summed effect on the measurement cell observation in the body frame.

Estimation of bias states enables localization corrections and their uncertainty can be modeled. In order to improve the observability of the ADCP sensor relative biases $\left(\mathbf{b}_{c, i}\right)$ and allow disambiguation from the true currents $\left(\mathbf{v}_{c, j}^{n}\right)$, rotation about heading is required, due to the transformation $\mathbf{C}_{n}^{b}$ in Equation 12 .

The ADCP bias with time can be modeled as a firstorder Markov process in a similar way to how a time varying, bounded bias can be modeled with IMU sensors (Flenniken IV (2005)):

$\dot{\mathbf{b}_{c, i}}=-\frac{1}{\tau_{\text {bias }}} \mathbf{b}_{c, i}+\nu_{\text {bias }}$

where $\tau_{\text {bias }}$ is the expected rate change of the ADCP sensor (a tuned parameter). $\nu_{\text {bias }}$ is a zero-mean normally distributed random variable with

$\sigma_{\text {bias }}=\sqrt{\frac{2 f \sigma_{\text {biasdrift }}^{2}}{\tau_{\text {bias }}}}$

where $\sigma_{\text {bias drift }}$ is the standard deviation of the bias in the long term, and limits the magnitude of the bias. It is a tuned parameter, which is given by the manufacturer as $1 \mathrm{~cm} / \mathrm{s}$. $f$ is the frequency at which the process model operates. $\tau_{\text {bias }}$ is a tuned parameter that can be be determined through accounting for the expected bias drift rate, which depends on a number of factors
Table 1 - Parameter values used in the 2DOF simulation

\begin{tabular}{|l|l|}
\hline GPS receiver & Lassen iQ GPS receiver \\
\hline Initial GPS position fix accuracy & $10 \mathrm{~m}\left(2 \sigma_{p}\right)$ \\
\hline Initial GPS velocity accuracy & $0.04 \mathrm{~m} / \mathrm{s}\left(2 \sigma_{v}\right)$ \\
\hline AUV descent rate & $0.2 \mathrm{~m} / \mathrm{s}$ \\
\hline ADCP make and model & RDI $1200 \mathrm{kHz}$ \\
\hline ADCP measurement uncertainty & $0.02 \mathrm{~m} / \mathrm{s}\left(2 \sigma_{a}\right)$ \\
\hline ADCP range & $30 \mathrm{~m}$ \\
\hline Water current depth cell size & $1 \mathrm{~m}$ \\
\hline Simulation time & $1000 \mathrm{~seconds}$ \\
\hline Simulated depth & $240 \mathrm{~m}$ \\
\hline DVL accuracy & $0.006 \mathrm{~m} / \mathrm{s}\left(2 \sigma_{D V L}\right)$ \\
\hline DVL range & $40 \mathrm{~m}$ \\
\hline DVL acquisition time & $1000 \mathrm{~seconds}$ \\
\hline ADCP and DVL update rate & $3 \mathrm{~Hz}$ \\
\hline Maximum currents & $20 \mathrm{~cm} / \mathrm{s}$ \\
\hline
\end{tabular}

as described previously. In this paper, a conservative time constant of 500 seconds is used and provided good performance on the experimental data.

\section{Vertical descent simulation}

This section explores the performance of the ADCP localization algorithm given vertical descent in simulation — first in two-dimensions and then in six-dimensions.

\subsection{Two degrees-of-freedom simulation}

The example in Section 3.1 and Figures 2(a) to 2(c) can be simulated to illustrate the operation and performance of the filter, including how the errors evolve in the states. This simulation illustrates how both Stanway's and this paper's method works. A 1-dimensional current field is simulated in which the vehicle is descending, and free to move left or right (but not into and out of the page). The vehicle experiences unmodeled drag (in the localization filter) which causes it to move with the currents. The vehicle is also assumed not to pitch resulting in two degrees-of-freedom (2DOF) in translation. To further simplify the analysis of this example, the bias states are not simulated nor estimated. Table 1 lists parameter values used for the 2DOF simulation.

To facilitate analysis, the full state history, or smoothed solution, of the information filter is used. All poses are kept in the state estimator. Figure 3(a) show the position of the ADCP-aided estimate before DVL bottomlock is obtained. The ADCP-aided estimate also has access to the ADCP observations. The ADCP-aiding filter position uncertainty growth is approximately $40 \mathrm{~m}$ $(2 \sigma)$. 


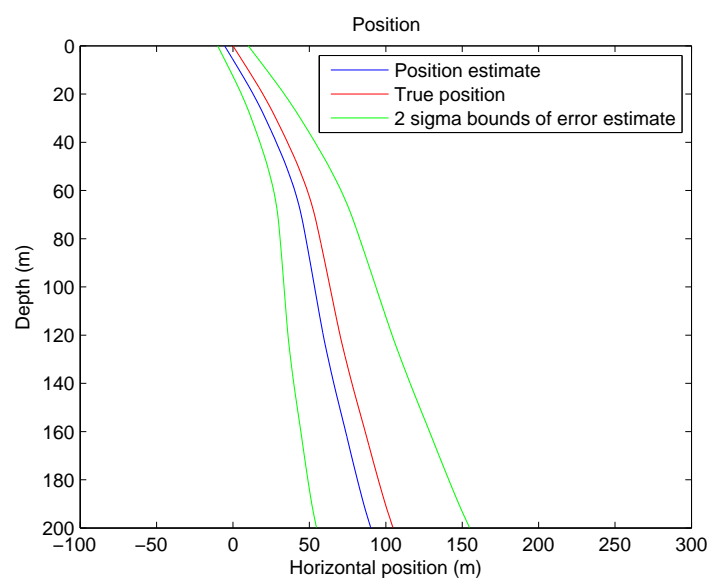

(a)

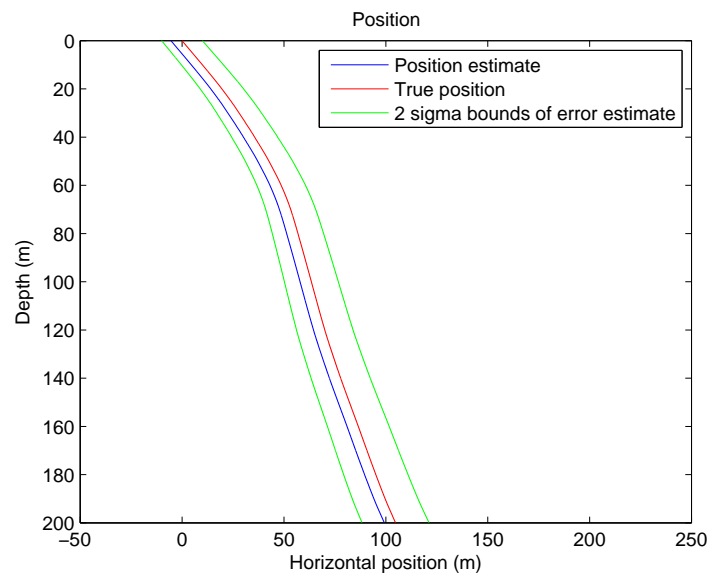

(b)

Fig. $3-2$ DOF simulation position estimates for the entire state history with the ADCP-aiding filter (a) before DVL bottom-lock and (b) after DVL bottom-lock.

Figure 3(b) show the position estimate after DVL bottom-lock is obtained for the entire state history. The DVL bottom-lock allows the entire velocity history to be constrained due to the correlations of vehicle velocity with water current velocity states, according to the ADCP measurements (Equation 12). The observation of DVL body-relative velocity is back-propagated to the entire descent because these correlations are accounted for in the Information Filter through the corrected water current estimates. The ADCP-aiding filter position uncertainty growth is now approximately $6 \mathrm{~m}(2 \sigma)$.

Figure 4(a) show the vehicle velocity errors before DVL bottom-lock for the entire state history. The velocity uncertainty slightly increases with time because of information loss from a finite number of uncertain measurements from the ADCP during the descent. Information loss is defined as the increase in velocity un- certainty as velocity estimates are extracted through re-observing the water currents with the ADCP, which translates into position uncertainty due to the position being the integral of velocity with time. This increase in velocity uncertainty is negligible because of the high number of re-observations of each current velocity bin (up to 750 times in this case). The uncertainty in velocity is primarily from the initial GPS velocity uncertainty, at $0.04 \mathrm{~m} / \mathrm{s}(2 \sigma)$.

While undergoing descent prior to DVL bottomlock, the velocity error is observed to have a Markov nature. The initial velocity error (from the surface) remains as a 'bias' in the vehicle velocity estimate during descent, since this error exists in the initial measured water currents. Thus, no matter how many subsequent measurements of the water currents are made while underwater without GPS, this initial velocity 'bias' in the water currents can never be overcome without another absolute velocity measurement.

Figures 4(b) show the velocity errors after DVL bottom-lock for the entire state history. The entire velocity history estimate uncertainty is $\sim 0.006 \mathrm{~m} / \mathrm{s}(2 \sigma)$, only slightly higher than the DVL accuracy, but for the entire dive. This slight increase is the result of the small information loss due to a finite number of uncertain measurements from the ADCP during the descent.

The absolute velocity measurement from the DVL allows the 'bias' in all of the water current measurements from the initial GPS velocity to be reduced in magnitude according to the uncertainty of the DVL. The errors and uncertainty bounds for the post-DVL bottom lock water current estimates are shown to be consistent in Figure 5(b). Applying this correction to all of the water currents reduces the previous uncertainty in the vehicle velocity. Figure 6 shows the estimated water currents from the filter post-DVL compared to the simulated ground truth, showing close overlap.

The 2DOF simulation has shown how the uncertainty evolves and illustrates that while the velocity uncertainty does increase in uncertainty during descent, the increase in uncertainty is very low relative to the initial velocity uncertainty. Once DVL bottom lock is acquired, the small increase in uncertainty of velocity applies backwards in time as well.

\subsection{Six degrees-of-freedom simulation}

This section extends the 2D simulation to a more complex and realistic six degrees-of-freedom (6DOF) AUV dive simulation with the following characteristics:

1. An initialization phase for the GPS/IMU is first simulated, using real output from a Novatel SPAN 


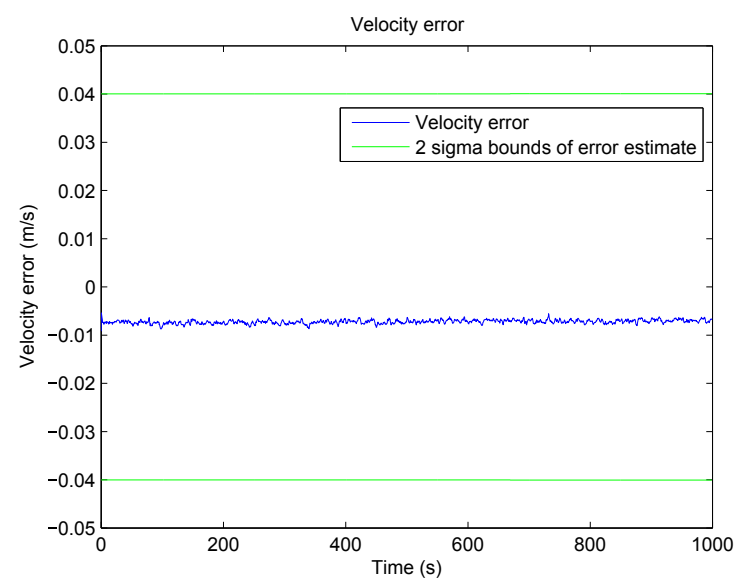

(a)

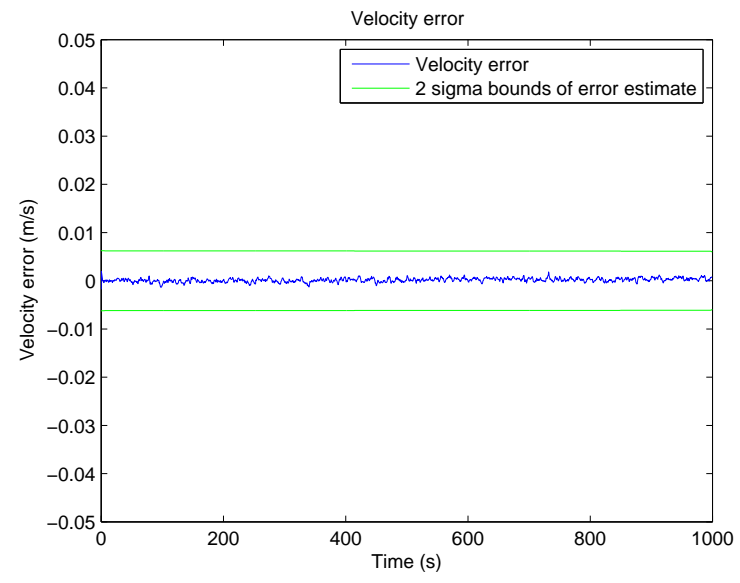

(b)

Fig. 4 - 2DOF simulation velocity errors for the entire state history with the ADCP-aiding filter (a) before DVL bottom-lock and (b) after DVL bottomlock.

system for realism. This allows the heading of the inertial system to be resolved. In this phase, Time Differenced Carrier Phase (TDCP) GPS is also available.

2. A vertical dive phase, where no GPS fix or DVL bottom-lock is available. The AUV rotates due to hydrodynamic forces, providing higher observability and thus enabling sensor bias estimation.

3. After one hour, the AUV acquires DVL bottom-lock at $40 \mathrm{~m}$ and starts to measure velocity over ground.

4. Following DVL bottom-lock, 30 seconds of DVL measurements are undertaken to allow velocity over ground to be estimated in combination with the IMU.

5. The water current velocities are correlated with depth, with a maximum current of $\sim 15 \mathrm{~cm} / \mathrm{s}$ - representative of typical ocean current profiles (Visbeck (2002)).

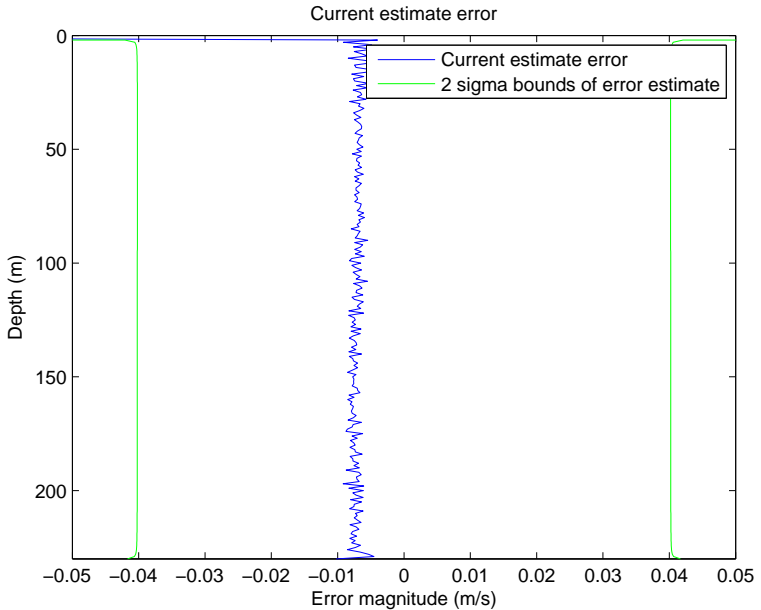

(a)

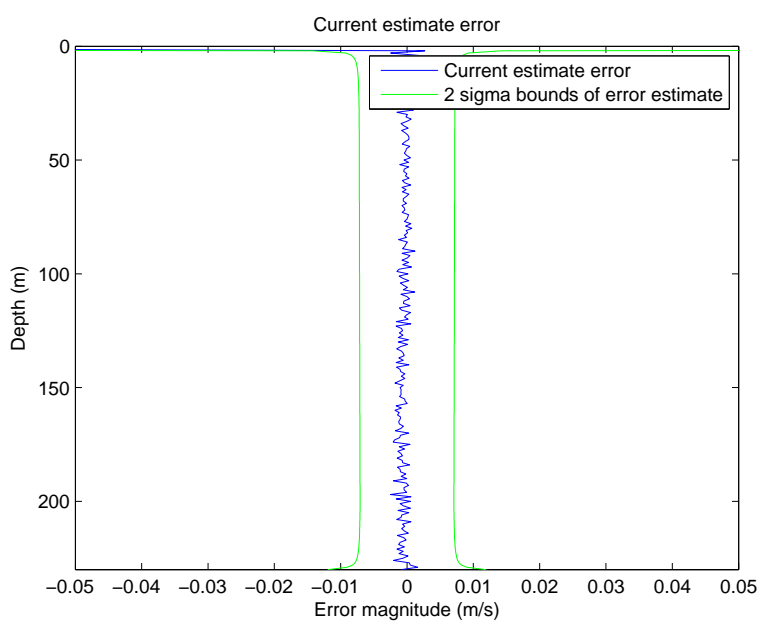

(b)

Fig. 5 - The error in the water current estimate from the 2DOF simulation (a) prior to DVL bottom-lock and (b) following DVL bottom lock.

This simulates a hypothetical mission undertaken by the Sirius AUV (Williams et al (2009)), where TDCP capable GPS is installed. Table 2 summarizes the parameter values used in the $6 \mathrm{DOF}$ simulation. In the subsequent simulation, the measurement cell bias magnitudes are constrained to zero within $1 \mathrm{~cm} / \mathrm{s}(2 \sigma)$, in alignment with the RDI specification (Gordon (1996)) and the calibration report on the RDI ADCP (Shih et al (2000)), which contains maximum biases of $\sim 1$ $\mathrm{cm} / \mathrm{s}$. The biases change with time in a correlated fashion (which accounts for changing depth during descent), simulating the bias effects described in section 3.2.1. A value for $\tau_{\text {bias }}$ in equation 13 of 500 seconds is used to simulate drifting biases over time scale, although controlled experiments of the ADCP sensor in real environments are required to identify a true value for this 


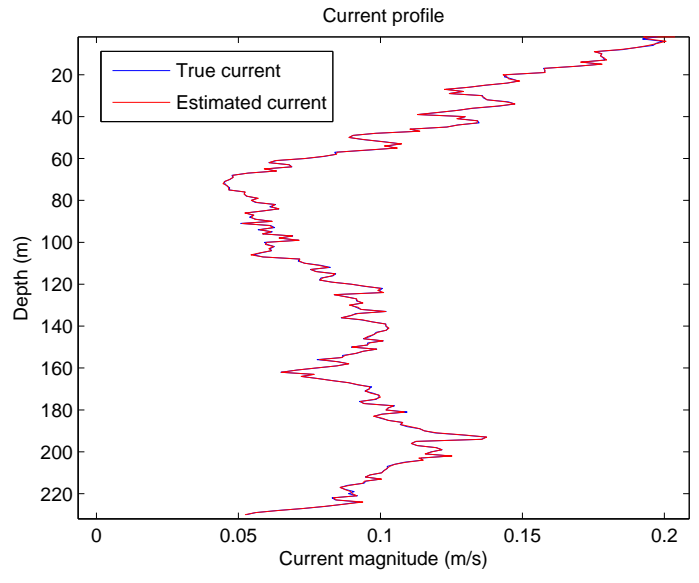

Fig. 6 - Current profile derived from the 2DOF simulation. Figure 5(b) shows the error plots.

Table 2 - Parameter values used in the $6 \mathrm{DOF}$ simulation.

\begin{tabular}{|l|l|}
\hline GPS receiver & Novatel SPAN \\
\hline Initial GPS position fix accuracy & $10 \mathrm{~m}\left(2 \sigma_{p}\right)$ \\
\hline Initial GPS velocity accuracy & $0.04 \mathrm{~m} / \mathrm{s}\left(2 \sigma_{v}\right)$ \\
\hline AUV descent rate & $0.2 \mathrm{~m} / \mathrm{s}$ \\
\hline ADCP make and model & RDI $1200 \mathrm{kHz}$ \\
\hline ADCP measurement uncertainty & $0.02 \mathrm{~m} / \mathrm{s}\left(2 \sigma_{a}\right)$ \\
\hline ADCP range & $30 \mathrm{~m}$ \\
\hline Water current depth cell size & $1 \mathrm{~m}$ \\
\hline Simulation time & 3605 seconds \\
\hline Simulated depth & $760 \mathrm{~m}$ \\
\hline DVL accuracy & $0.006 \mathrm{~m} / \mathrm{s}\left(2 \sigma_{D V L}\right)$ \\
\hline DVL range & $40 \mathrm{~m}$ \\
\hline DVL acquisition time & 3600 seconds \\
\hline ADCP and DVL update rate & $3 \mathrm{~Hz}$ \\
\hline Maximum currents & $20 \mathrm{~cm} / \mathrm{s}$ \\
\hline IMU & Honeywell HG1700A58 \\
\hline IMU bias stability & $1 \mathrm{degree} / \mathrm{hour}$ \\
\hline AUV rotation rate & $8 \mathrm{degrees} / \mathrm{second}$ \\
\hline Bias magnitude $\left(\sigma_{\text {bias drift }}\right)$ & $0.01 \mathrm{~m} / \mathrm{s}(2 \sigma)$ \\
\hline Time constant of bias $\left(\tau_{\text {bias }}\right)$ & $500 \mathrm{~seconds}$ \\
\hline TDCP GPS uncertainty & $5 \mathrm{~mm}(2 \sigma)$ \\
\hline TDCP GPS rate & $2 \mathrm{~Hz}$ \\
\hline
\end{tabular}

parameter. This bias estimation is unique to our work and is not performed in Stanway's implementation.

A tactical-grade Honeywell HG1700A58 IMU was simulated, providing position, velocity and attitude constraints through the integration of the body rotation rates and accelerations (incorporating IMU constraints was not undertaken in Stanway's implementation). The method used to incorporate the inertial measurements into the filter is based on Lupton and Sukkarieh (2009) and Lupton (2010). A global reference frame is used, and initial attitude is assumed accurate for linearization purposes. Compensation for Earth rotation (significant for heading estimation during long missions), as calcu- lated in Titterton and Weston (2004), is achieved with the following equation, which is modified from Algorithm 1, Line 9 in Lupton and Sukkarieh (2009):

$\Delta \phi_{\mathbf{t}+\mathbf{1}}=\boldsymbol{\Delta} \phi_{\mathbf{t}}+\mathbf{E}_{\mathbf{t}}^{\mathbf{t 1}}\left(\omega_{\mathbf{t}}^{\mathrm{b}}-\operatorname{bias}_{\mathrm{gyro}}-\mathbf{C}_{\mathbf{n}}^{\mathrm{k}} \Omega_{\mathrm{e}}^{\mathbf{n}}\right) \Delta t$

where $-\mathbf{C}_{\mathbf{n}}^{\mathbf{k}} \boldsymbol{\Omega}_{\mathbf{e}}^{\mathbf{n}}$ is the apparent Earth rotation in the body frame. The gyro and accelerometer bias states are augmented into the filter. As illustrated in section 4.1, the error growth in position before DVL bottom-lock is dependent on the initial velocity uncertainty while on the surface. A typical velocity uncertainty of $4-8 \mathrm{~mm} / \mathrm{s}$ $(2 \sigma)$ in the horizontal directions is possible with standard GPS by exploiting the carrier phase on the GPS receiver (van Graas and Soloviev (2004)). This means that during descent, position error growth can also be constrained to the $4-8 \mathrm{~mm} / \mathrm{s}(2 \sigma)$ range, which is similar to DVL velocity uncertainty.

TDCP (Soon et al (2008)) is a particular implementation of carrier phase processing. It can be approximately modeled as tracking the change in position of the vehicle (while on the surface).

$$
\begin{aligned}
h_{T D C P}\left(\hat{\mathbf{x}}\left(t_{k}\right)\right) & =\mathbf{p}^{n}\left(t_{k}\right)-\mathbf{p}^{n}\left(t_{k-1}\right) \\
R_{T D C P} & =\sigma_{T D C P}^{2}
\end{aligned}
$$

The TDCP observation is dissimilar to the DVL observation, as it is a change-in-position measurement rather than an instantaneous velocity measurement. The delayed state structure of the EIF allows this observation between two poses to be correctly accounted for (Soon et al (2008)). The TDCP change-in-position measurement is assumed to have an uncertainty of $5 \mathrm{~mm}(2 \sigma)$ occurring at $2 \mathrm{~Hz}$. In Stanway's implementation, TDCP GPS was not considered.

\subsubsection{AUV dynamic vehicle modeling}

For the simulation, we assume that the vehicle has differential thrust to control heading and forward velocity (Fossen (1994)). This information is used to generate the simulated true motion of the vehicle given vertical thrust through the water column. Within the filter, a dynamic vehicle model is not used as it is beyond the scope of this paper. Pitch and roll are set to zero in the simulation, although the filter estimates these states. The vehicle model applies the following state equations:

$\mathbf{M} \dot{\mathbf{v}}+\mathbf{C}(\mathbf{v}) \mathbf{v}+\mathbf{D}(\mathbf{v}) \mathbf{v}+\mathbf{C}_{n}^{b}\left(m \mathbf{g}^{n}+\mathbf{b}^{n}\right)=\boldsymbol{\tau}$ 
Table 3 - Vehicle model parameters used in the 6 DOF simulation.

\begin{tabular}{|l|l|}
\hline Symbol & True value \\
\hline$M_{x}$ & $500 \mathrm{~kg}$ \\
\hline$M_{y}$ & $500 \mathrm{~kg}$ \\
\hline$M_{z}$ & $225 \mathrm{~kg}$ \\
\hline$I_{z}$ & $179.049 \mathrm{~kg} \mathrm{~m}^{2}$ \\
\hline$D_{\dot{x}}$ & $500 \mathrm{~kg} \mathrm{~m}^{-1}$ \\
\hline$D_{\dot{y}}$ & $800 \mathrm{~kg} \mathrm{~m}^{-1}$ \\
\hline$r$ & $0.2 \mathrm{~m}$ \\
\hline
\end{tabular}

$$
\begin{array}{r}
\mathbf{v}=\left[\begin{array}{c}
\dot{\dot{z}_{b}} \\
\dot{y_{b}} \\
\dot{z_{b}} \\
\dot{\psi}
\end{array}\right] \quad(19) \boldsymbol{\tau}=\left[\begin{array}{c}
F_{1}+F_{2} \\
0 \\
F_{3} \\
F_{1} r-F_{2} r
\end{array}\right] \\
\mathbf{M}=\operatorname{diag}\left\{M_{x}, M_{y}, M_{z}, I_{z}\right\} \\
\mathbf{C}(\mathbf{v})=\left[\begin{array}{cccc}
0 & 0 & 0-M_{y} \dot{y_{b}} \\
0 & 0 & 0 & M_{x} \dot{x_{b}} \\
0 & 0 & 0 & 0 \\
M_{y} \dot{y_{b}}-M_{x} \dot{x_{b}} & 0 & 0
\end{array}\right]
\end{array}
$$

$$
\mathbf{D}(\mathbf{v})=-\operatorname{diag}\left\{D_{\dot{x}}\left|\dot{x_{b}}\right|, D_{\dot{y}}\left|\dot{y}_{b}\right|, D_{\dot{z}}\left|\dot{z}_{b}\right|, D_{\dot{\psi}}|\dot{\psi}|\right\}
$$

where

$-\dot{x_{b}}, \dot{y_{b}}$ and $\dot{z_{b}}$ are the water relative velocities of the vehicle in the body frame in the forward, starboard and down directions respectively.

- $\dot{\psi}$ is the yaw rotational velocity of the vehicle

- $\mathbf{M}$ is the inertia matrix (including added mass)

- $\mathbf{C}(\mathbf{v})$ is the matrix of Coriolis and centripetal terms (including added mass)

- $\mathbf{D}(\mathbf{v})$ is the damping matrix

- $\tau$ is the vector of control inputs

- $F_{1}, F_{2}$ and $F_{3}$ are the thrusts from the port, starboard and vertical thruster respectively

- $m$ is the true mass of the vehicle

- $\mathbf{g}^{n}$ is the gravity vector in the navigation frame

$-\mathbf{b}^{n}$ is the buoyancy force in the navigation frame

- $r$ is the distance from the thruster to the center of mass along the perpendicular direction of the applied force.

Vehicle parameters are estimated using Martin and Whitcomb (2008) as a baseline, and listed in Table 3.

Additionally, a thruster model according to Healey et al (1995) and Fossen (1994) is utilized:

$F=0.4 \rho d^{4}|n| n-\frac{1}{3} v_{T} \rho d^{3}|n|$

where

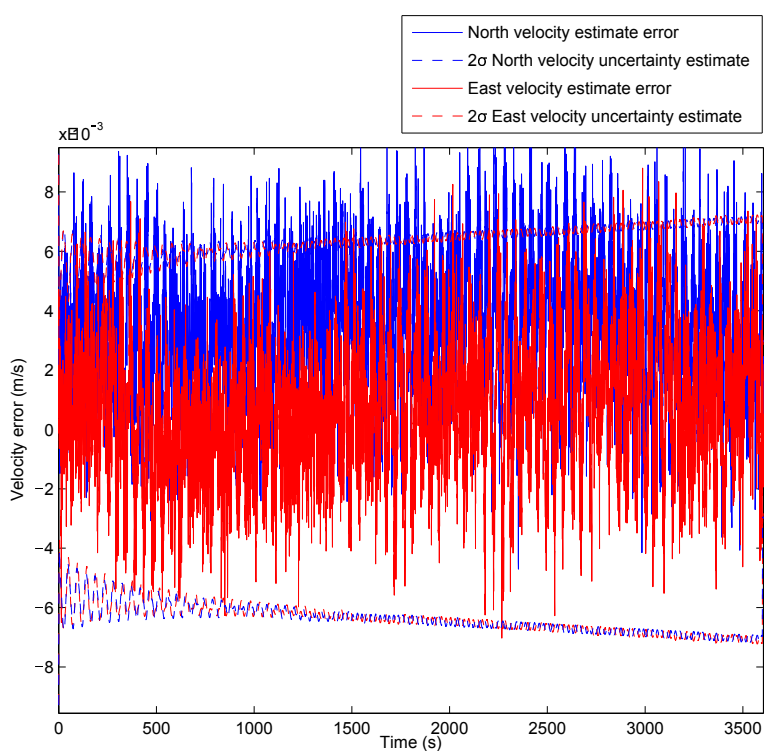

(a)

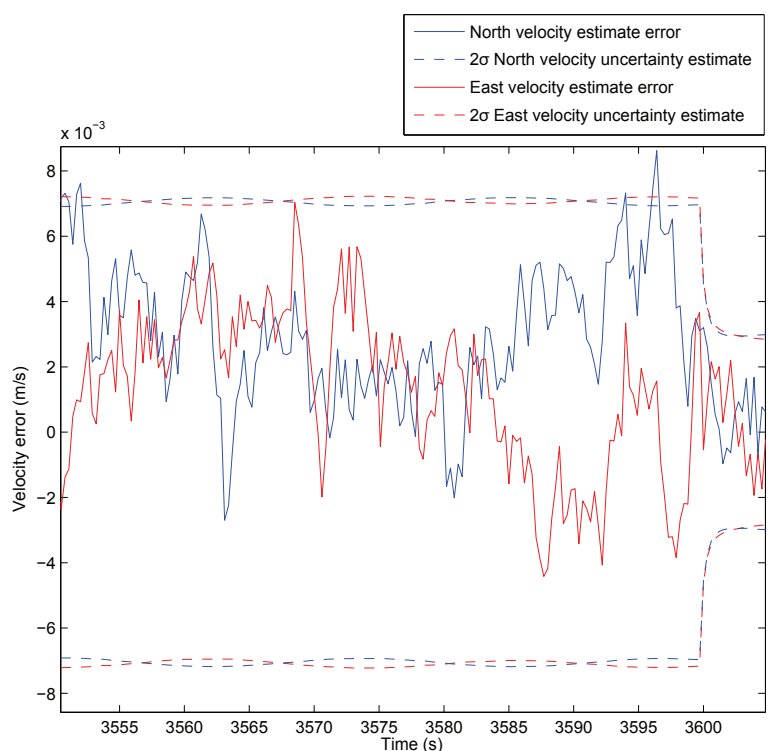

(b)

Fig. 7 - Sirius simulation (a) run-time filter velocity $2 \sigma$ uncertainties with time. (b) An enlarged detail on the effect of the DVL bottom-lock on the velocity estimates at 3600 seconds.

$-\rho$ is the density of water

$-d$ is the diameter of the propeller

$-n$ is the revolution speed of the thruster

$-v_{T}$ is the velocity of the water going into the propeller 


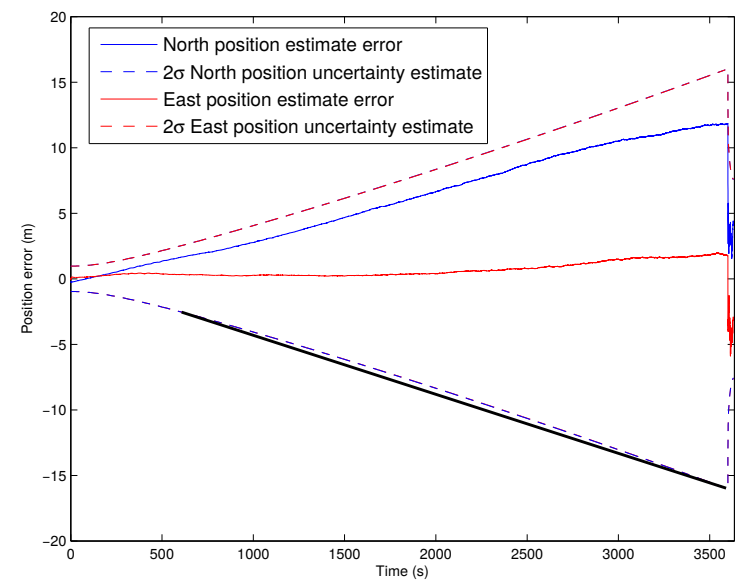

(a)

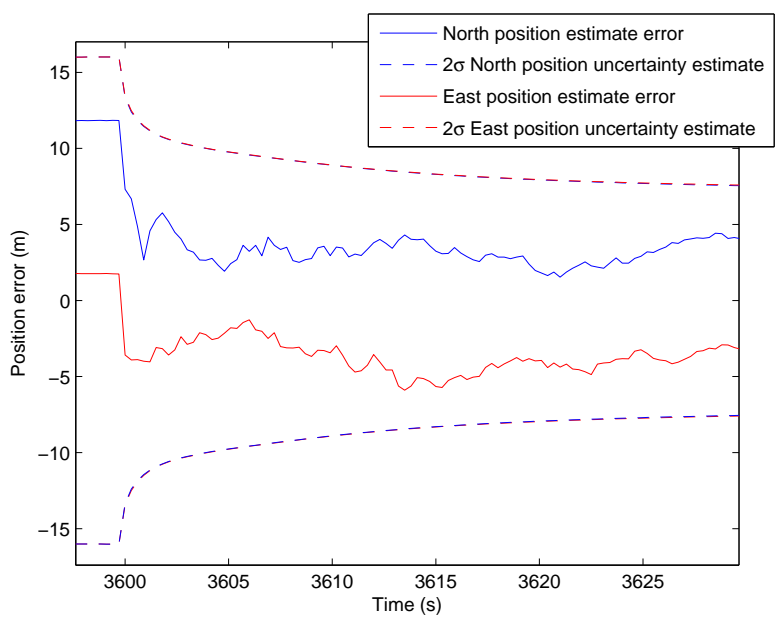

(b)

Fig. 8 - Sirius simulation (a) run-time filter position $2 \sigma$ uncertainty with time. A black line is drawn for a portion of the position uncertainty bounds to illustrate the slight non-linearity of the uncertainty growth. (b) An enlarged detail on the effect of the DVL bottom-lock on the position estimate at 3600 seconds.

\subsubsection{Sirius simulation results for one hour descent}

The increasing computational complexity with time necessary to perform smoothing and maintain the full pose history requires that we marginalize of all pose states in the filter except those for the present and previous time step. This was applied to provide run-time pose estimates and uncertainties, effectively creating a delayedstate EKF. For constant-time complexity in the filter, we marginalized water column states which will not be re-observed. Thus, this filter implementation has the potential to be implemented in real-time on a vehicle.
This same strategy is applied in Section 6 for the realworld deep-water missions.

Figure 7 shows the run-time filter uncertainty estimate of velocity is constrained to $\sim 7 \mathrm{~mm} / \mathrm{s}(2 \sigma)$ in the north and east directions. This uncertainty in velocity during the dive phase is from the initial velocity uncertainty after GPS/IMU initialization and ADCP estimation on the surface, including TDCP. The initial velocity estimate in the north direction on the surface was near the boundary of the $2 \sigma$ uncertainty estimate. Thus, it will continue to be on the boundary, with the noisy north velocity estimate error exceeding the upper $2 \sigma$ bounds. This is normal behavior for the filter as a result of this initial velocity estimate. During descent prior to DVL bottom-lock, the velocity error is observed to have a Markov nature. The initial velocity error (from the surface) remains as a 'bias' in the vehicle velocity estimate during descent since this error exists in the initial measured water currents. This is similar to the behavior observed in Section 4.1. In this case, over the 3600 s time span, the velocity uncertainty increases slightly, implying that some information loss occurs from the continual initialization of new water current depth cells.

As shown in Figure 8(a), just prior to DVL bottomlock after $3600 \mathrm{~s}$, the position uncertainty estimate is $\sim 16 \mathrm{~m}(2 \sigma)$ in the north and east directions, or $15 \mathrm{~m}$ uncertainty growth compared to the surface uncertainty. Figure 8(b) shows an enlarged detail of the effect of DVL bottom-lock on the position estimates, with the $2 \sigma$ position estimate uncertainty reaching $\sim 7.5 \mathrm{~m}$, or 6.5 $\mathrm{m}$ uncertainty growth from the surface uncertainty of 1 $\mathrm{m}$. The position estimates show a correlated, but consistent, error with respect to the $2 \sigma$ uncertainty bounds. This shows that the north velocity estimates, with errors exceeding their $2 \sigma$ uncertainty bounds, once integrated through the IMU model produce the expected, consistent result for position uncertainty. The velocity uncertainty does not necessarily equal the position uncertainty growth rate, as a result of the non-linear IMU integration and the velocity estimate interacting with the ADCP bias estimation with rotation. This results in non-trivial correlation between the position and velocity estimates, captured through the information matrix.

Figure 9 shows the performance for USBL, LBL, IMU/vehicle model/DVL water-track, and our reported method for a one hour descent to the seafloor. $(2 \sigma)$ for USBL and LBL are relatively constant and are $\sim 10 \mathrm{~m}$. The IMU/vehicle model/DVL water-track navigation solution (extrapolated from available results) increases with time and has an error of $\sim 120 \mathrm{~m}$ after 1 hour. In comparison, our method accumulates less than $20 \mathrm{~m}$ error during the descent and, after DVL bottom-lock, 


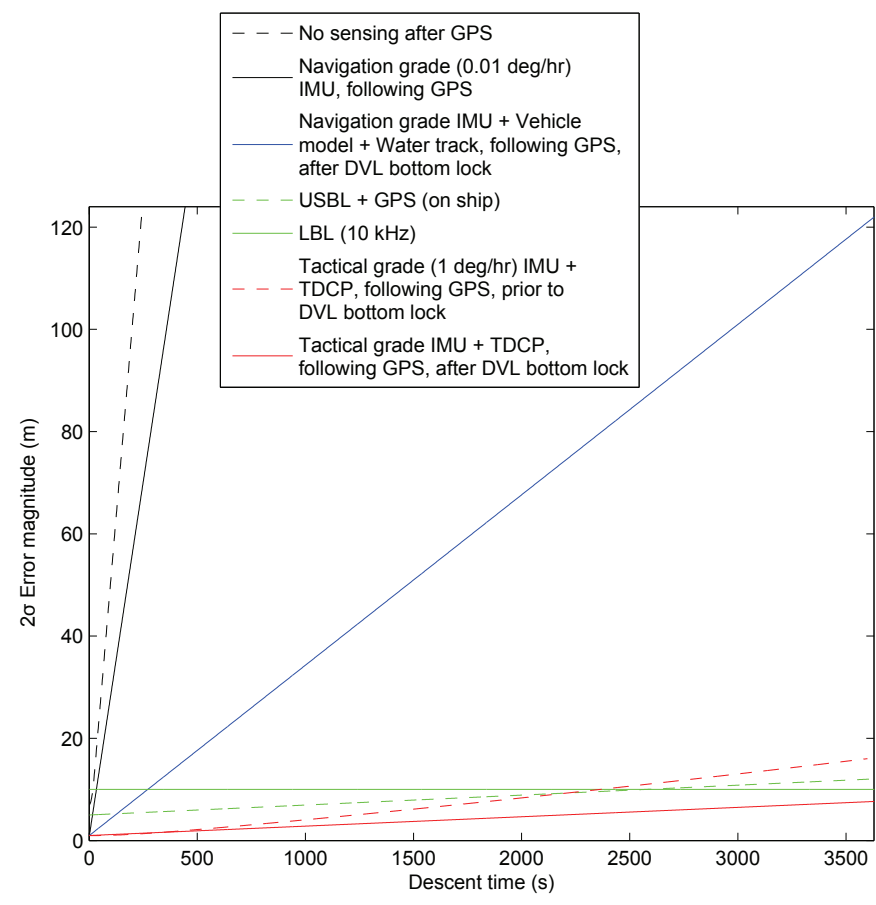

Fig. 9 - Underwater localization methods and their approximate accuracy for a 1 hour descent. USBL and LBL solutions are shown in green. Blue is a IMU/vehicle model/DVL water-track navigation solution obtained by extrapolating available results. The reported algorithm performance is shown in red. The new localization method compares favorably in this case with acoustic localization methods, and outperforms existing selfcontained techniques.

the error decreases to less than $10 \mathrm{~m}$. This demonstrates the ability of our method to provide accuracy comparable to the acoustic localization methods but without external infrastructure and at a higher update rate.

\section{Experiments with the Sirius AUV and View-based SLAM}

Initial field experiments were obtained with the Sirius AUV, a modified version of the mid-sized SeaBED AUV(Singh et al (2004b)), owned and operated by the University of Sydney Australian Centre for Field Robotics (ACFR) (Williams et al (2009)). Designed for highresolution imaging, the AUV is passively stable in roll and pitch. Navigation sensors include a $1200 \mathrm{kHz}$ RDI DVL/ADCP, Tracklink 1500 HA USBL and a Lassen iQ GPS receiver. Additionally, a stereo imaging platform allows for six degree-of freedom view-based loop closures (Mahon et al (2008); Williams et al (2010)) when near the seafloor (typically $\sim 2 \mathrm{~m}$ altitude). These experiments employ a constant velocity process model

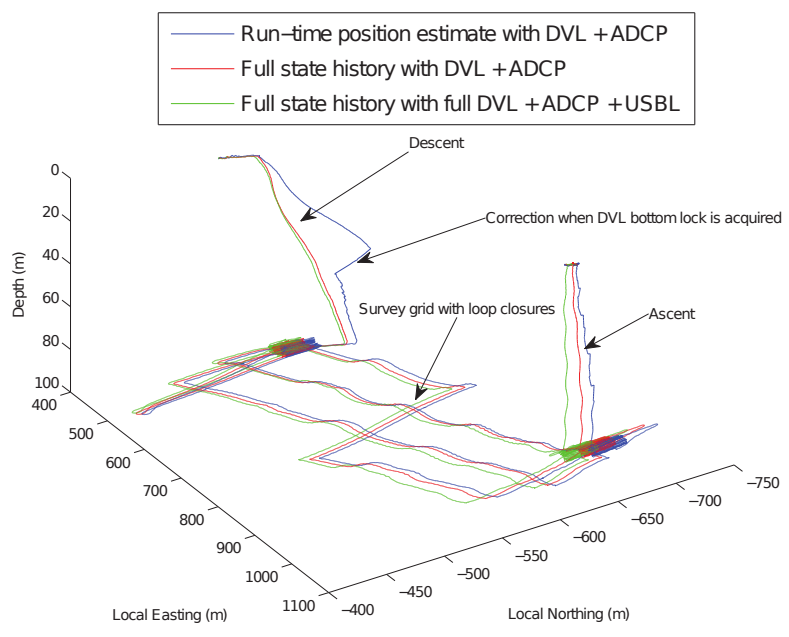

(a)

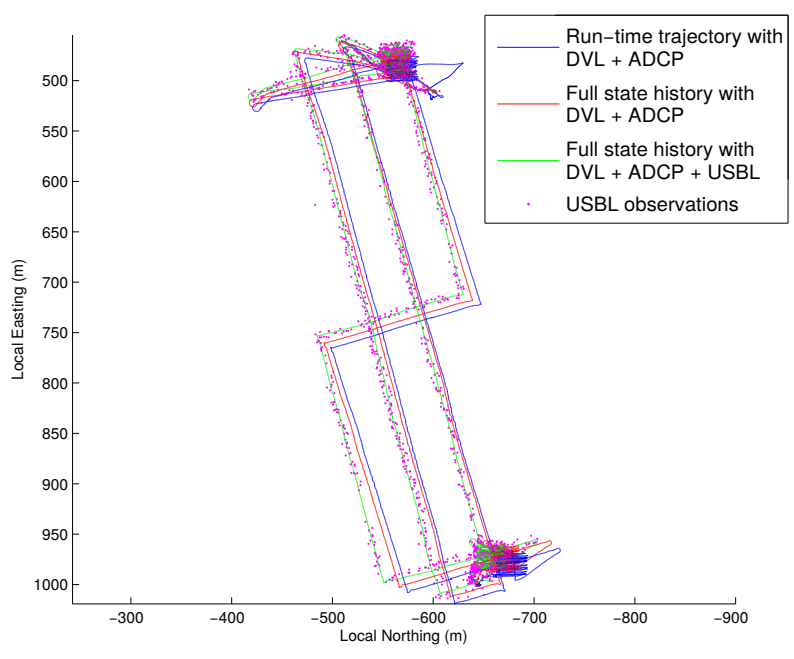

(b)

Fig. 10 - (a) Oblique and (b) Bird's eye view of the trajectory for the Sirius Freycinet mission, where DVL bottom-lock is only available at $40 \mathrm{~m}$ altitude.

tuned for the most extreme dynamics of the vehicle. Attitude information is supplied by the in-built magnetic compass and roll/pitch tilt sensors in the RDI DVL/ADCP. These results were previously reported in Medagoda et al (2011).

Experimental data is from a 2010 mission on coastal reefs near the Freycinet Peninsula in eastern Tasmania (Williams et al (2012)) in which Sirius completed a 3 hour dive to $100 \mathrm{~m}$ depth. DVL bottom-lock was unavailable during descent and ascent. Ground truth measurements are provided by USBL; position estimates are shown in Figure 10. Additional information and data from another site are reported in Medagoda et al (2011). 
The full state history, or smoothed solution, of the filter is defined as the output of the EIF at the end of the mission. All of the poses are maintained by the estimator. The run-time filter is the maximum-likelihood estimate of pose of the vehicle at that instance in the mission. Results in Figure 11(a) show how the ADCP method, without the USBL, results in georeferencing for the subsequent seafloor view-based SLAM-aided mission. Georeferencing uncertainty is within $20 \mathrm{~m}(2 \sigma)$ position accuracy while the AUV is near the seafloor, and after post-ascent GPS acquisition, the accuracy of the seafloor portion is within $11 \mathrm{~m}(2 \sigma)$. The ADCP was interleaved with the DVL at a ratio of $1: 5$, with the ADCP operating at approximately $0.5 \mathrm{~Hz}$. Despite low ADCP update rates, it is possible to localize without an external acoustic source (e.g., USBL). The ascent and descent water current velocity states differ because of both temporal (i.e., a 3 hour time interval between the descent and ascent) and spatial (i.e., $\sim 600 \mathrm{~m}$ horizontal distance) changes.

Figure 11(a) also shows the ability of SLAM loopclosure to improve the ADCP-aided navigation. It shows that the method can be improved with imagery due to loop closures, but that the entire mission can be localized without them. Figure 11(a) shows the effect of loop closures at $\sim 3500 \mathrm{~s}$ into the mission - image feature matching identified that the AUV has revisited a previous site in the mission resulting in a realtime decrease in uncertainty for the filter. This and subsequent loop closure observations constrain the position uncertainty during the seafloor mapping portion of the dive, thus providing significantly improved localization for the seafloor portion of the mission. This occurs after the post-ascent GPS acquisition, due to the increased correlation of temporally distant poses, as seen in Figure 11(a).

Figure 11(b) compares the filter result with the independent USBL observations. During the descent, prior to DVL bottom-lock, the realtime filter is inconsistent. Once DVL bottom-lock is acquired, relinearization occurs with the EIF and the error is reduced, providing evidence that the previously observed inconsistency was due to linearization error. The relinearization was achieved by calculating Jacobians for previous measurements with the present estimate of that state, and then re-constructing the information vector and matrix and solving for the state. These equations are shared or modified from Equation 4, 9, 10 and 11.

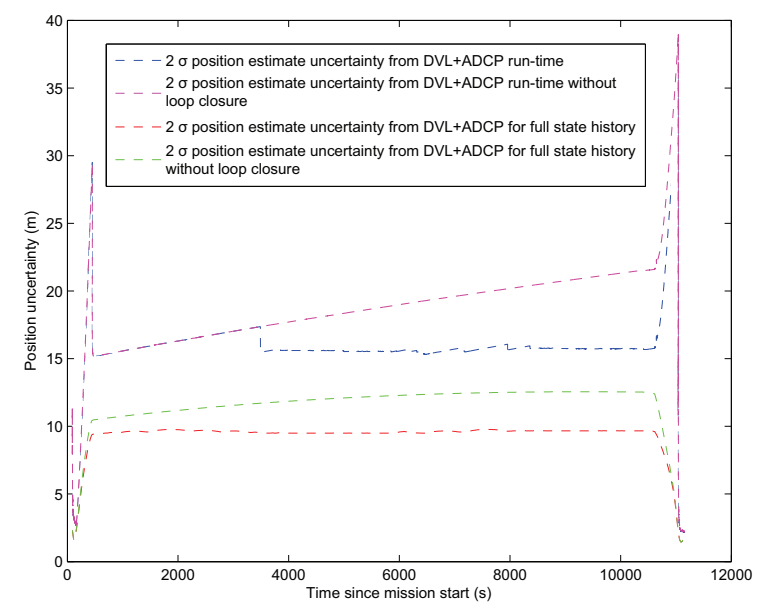

(a)

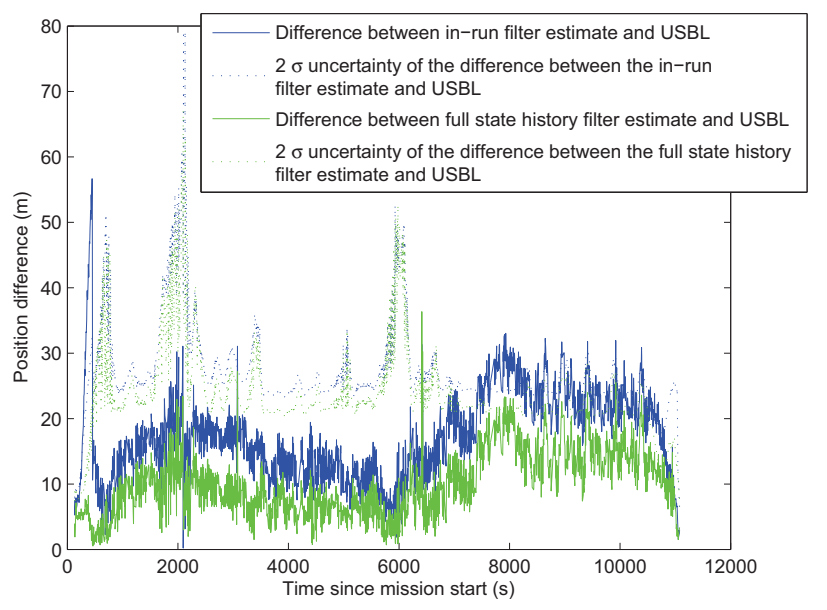

(b)

Fig. 11 - (a) Sirius Freycinet position uncertainty estimates for filter in-run and for the full state history, with and without loop closures (b) Differences between filter results and USBL measurements, along with $2 \sigma$ uncertainties of this difference, showing that the filter is consistent after DVL bottom-lock.

$$
\begin{aligned}
\hat{\mathbf{y}}^{+}\left(t_{e}\right) & =\hat{\mathbf{y}}^{-}\left(t_{0}\right)+\sum_{t_{n}=t_{0}}^{t_{e}} \mathbf{i}\left(t_{n}\right) \\
\hat{\mathbf{Y}}^{+}\left(t_{e}\right) & =\hat{\mathbf{Y}}^{-}\left(t_{0}\right)+\sum_{t_{n}=t_{0}}^{t_{e}} \mathbf{I}\left(t_{n}\right) \\
\hat{\mathbf{y}}^{+}\left(t_{e}\right) & =\hat{\mathbf{Y}}^{+}\left(t_{e}\right) \hat{\mathbf{x}}^{+}\left(t_{e}\right)
\end{aligned}
$$

in which

$$
\begin{aligned}
\mathbf{i}\left(t_{n}\right)= & \nabla_{x}^{\top} \mathbf{h}\left(t_{e}\right) \mathbf{R}^{-1}\left(t_{n}\right)\left(\mathbf{z}\left(t_{n}\right) \ldots\right. \\
& \left.-\mathbf{h}\left(\hat{\mathbf{x}}^{-}\left(t_{e}\right)\right)+\nabla_{x} \mathbf{h}\left(t_{e}\right) \hat{\mathbf{x}}^{-}\left(t_{e}\right)\right) \\
\mathbf{I}\left(t_{n}\right)= & \nabla_{x}^{\top} \mathbf{h}\left(t_{e}\right) \mathbf{R}^{-1}\left(t_{n}\right) \nabla_{x} \mathbf{h}\left(t_{e}\right)
\end{aligned}
$$


where $t_{n}$ is a measurement time between the be ginning of the descent $\left(t_{0}\right)$ to the end of the descen $\left(t_{e}\right)$. This strategy is applied on ascent as well, substi tuting the beginning of the ascent for $t_{0}$ and the enc of the ascent for $t_{e}$. We observe that the ADCP filter is consistent with the USBL observations, validating the performance of the ADCP-aiding method. The above results show how ADCP-aided navigation during the descent and ascent of a mission, coupled with viewbased SLAM on the seafloor, allows georeferencing even with infrequent ADCP measurements.

\section{Deep water Experiments with the Sentry AUV}

Our ADCP-aided localization algorithm was validated in deep water environments using data obtained with the Sentry AUV. Sentry is a $6000 \mathrm{~m}$ rated AUV designed and built by Woods Hole Oceanographic Institution (WHOI) for geophysical, geochemical, and biological surveys and is operated by WHOI for the U.S. scientific community (Kinsey et al (2011)). The ADCP sensor is a $300 \mathrm{kHz}$ RDI Navigator with $120 \mathrm{~m}$ maximum range with default settings, although with reduced accuracy compared to the $1200 \mathrm{kHz}$ sensor on the Sirius AUV. The process model used for the vehicle is a constant velocity model. The process noise is tuned to worst case vehicle dynamics and no thruster model is incorporated. Depth information is supplied by a Paroscientific depth sensor and USBL measurements by a Sonardyne USBL system. Attitude information is supplied by an Ixsea Phins INS gyrocompass; however raw IMU measurements of acceleration and rotation rates were not available. Our method is tested on three dives - Sentry dive 220 in December 2013 and Sentry dives 273 and 281 in July 2014.

The dive site for 220 was at the Dorado Outcrop, located west of Costa Rica in the Pacific Ocean. On this deployment, Sentry dives to $\sim 3 \mathrm{~km}$ in over 6000 $\mathrm{s}$, with a descent rate of $\sim 0.4 \mathrm{~m} / \mathrm{s}$. Initial velocity and position is estimated using the USBL for the first $500 \mathrm{~s}$ of the descent, as the vehicle was not equipped with GPS. During $5354 \mathrm{~s}$ of descent, the vehicle does not have DVL bottom lock and the USBL undergoes a simulated blackout to illustrate the ADCP-aided filter. The USBL serves as ground truth. The ADCP was run open-loop interleaving 1:1 with the DVL.

For computational efficiency, the marginalized EIF is used from the Sirius simulation. Imagery was not available; thus view-based SLAM is not incorporated. During this descent, a high amount of ADCP data errors were detected, as shown in Figure 12, reducing the effective ADCP rate to $1.9 \mathrm{~Hz}$. The error metrics used

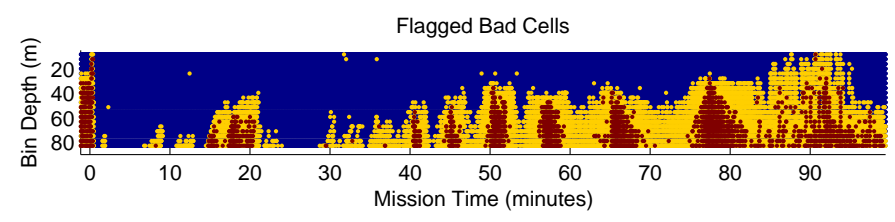

Fig. 12 - Data errors in the ADCP sensor for Sentry 220. The data in blue is good data prior to the $\chi^{2}$ test. The data in maroon has failed the percent good self-diagnostic of the sensor. The data in yellow exceeds the error velocity threshold.

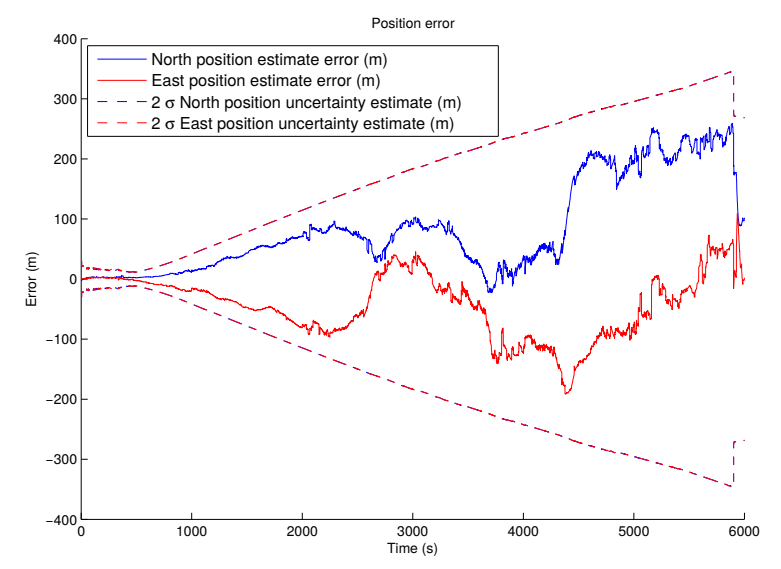

Fig. 13 - Sentry 220 position errors and uncertainty estimates for the filter. The filter is consistent as the errors are within the $2 \sigma$ bounds for the uncertainty estimates. The error is the difference between the estimate and the USBL ground truth.

to reject measurements are an error velocity threshold, percent good reported from sensor, and $\chi^{2}$ test for normalized innovations. The cause of these data errors are unknown and resolving them remains as future work. The rejected measurements results in the correlations being reduced throughout the descent.

Figure 13 shows the position error and uncertainty estimates during the descent. DVL bottom-lock results in a velocity and position correction once the altitude is below approximately $240 \mathrm{~m}$. The position uncertainty growth is $328 \mathrm{~m}(2 \sigma)$ prior to DVL bottom lock, giving an position error growth rate of approximately 6.1 $\mathrm{cm} / \mathrm{s}(2 \sigma)$. The velocity uncertainty during the descent is plotted in Figure 14. The velocity uncertainty following the combined USBL/ADCP initialization is approximately $7.5 \mathrm{~cm} / \mathrm{s}(2 \sigma)$. Thus the position growth rate and velocity uncertainty are of similar magnitude, with differences due to the estimation of ADCP biases and the Markov nature of the estimation as discussed in Section 4.2.2. Once DVL bottom lock is acquired, the position uncertainty growth is $\sim 254 \mathrm{~m}(2 \sigma)$, with a po- 


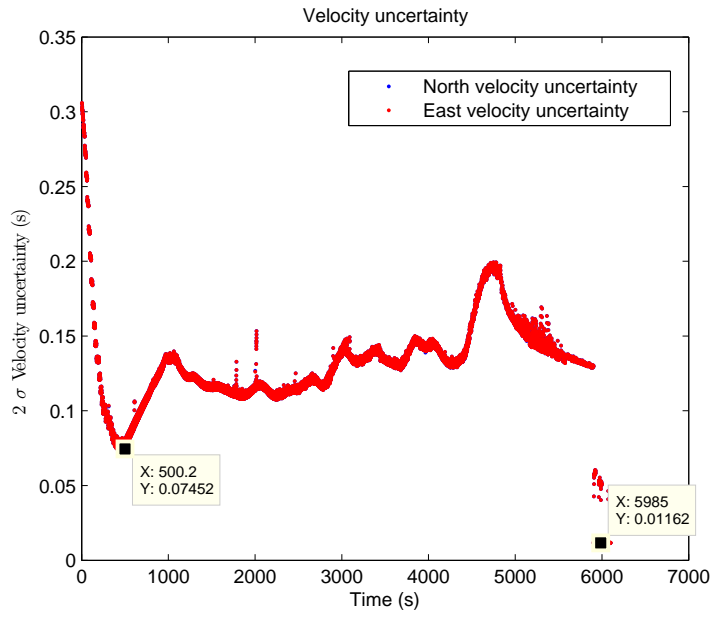

Fig. 14 - Sentry 220 North and East velocity uncertainty of the filter. The red east velocity uncertainty mostly overlaps with the blue velocity uncertainty. Increases in the velocity uncertainty during decent are due to data errors which result in rejected measurements.

sition uncertainty growth rate of $4.7 \mathrm{~cm} / \mathrm{s}(2 \sigma)$ while the velocity uncertainty is reduced to $1.1 \mathrm{~cm} / \mathrm{s}(2 \sigma)$ due to the DVL sensor. This uncertainty reduction once DVL bottom lock is acquired is less than what was possible in simulation in Section 4.2.2, due to the high amount of ADCP data errors resulting in reduced correlation during the descent and the lack of IMU data .

The velocity uncertainty estimates in Figure 14 also increase as ADCP measurements are rejected more often, corresponding to the errors identified in Figure 12. Figure 15 shows the position estimates compared to the USBL position measurements. Over the $3 \mathrm{~km}$ descent a $\sim 350$ m westerly drift is experienced because of water currents. The final error in the position estimation was $88 \mathrm{~m}$, showing an advantage compared to a zero drift assumption.

Sentry dives 273 and 281 were completed off the coast of Oregon at the ASHES vent site, where Sentry descends to approximately $1500 \mathrm{~m}$. The descent rate is $\sim 0.6 \mathrm{~m} / \mathrm{s}$, higher than Sentry 220 mission due to different ballasting. Initial velocity and position is estimated using the USBL for the first portion of the descent until the uncertainty in velocity is $7.5 \mathrm{~cm} / \mathrm{s}$, allowing comparisons to the Sentry 220 mission. During the descent, the vehicle does not have DVL bottom lock and the USBL undergoes a simulated blackout to illustrate the ADCP-aided filter. Again, USBL serves as the ground truth.

The marginalized EIF is again utilized and viewbased SLAM is not incorporated. Figures 16 and 18

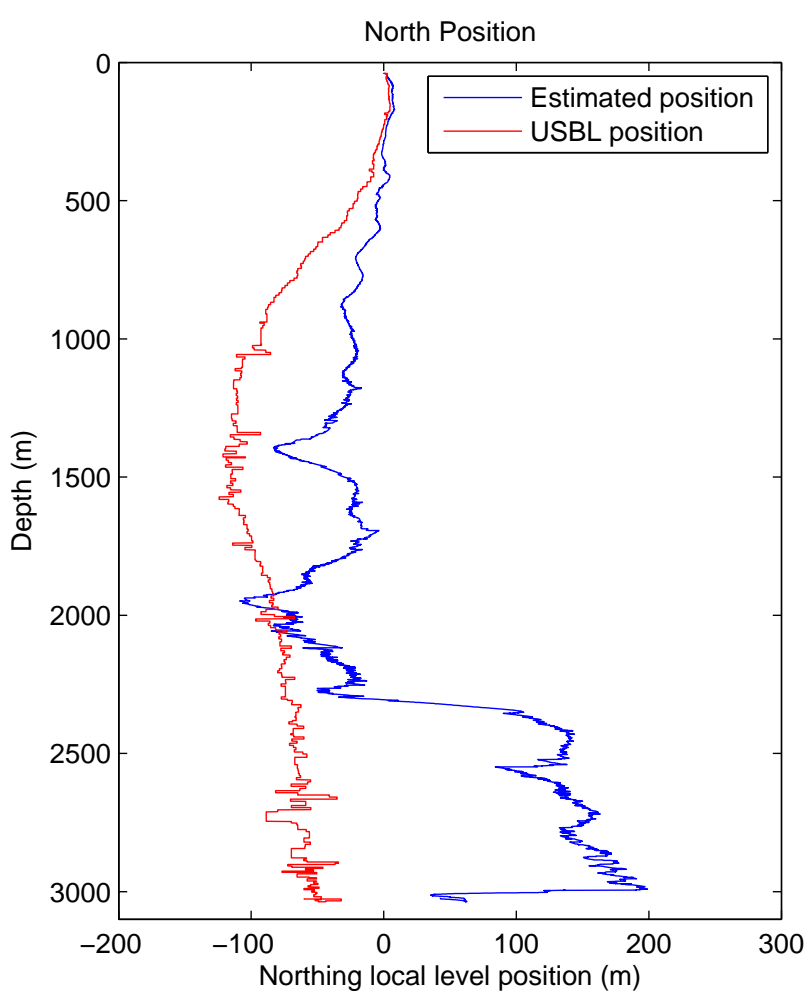

(a)

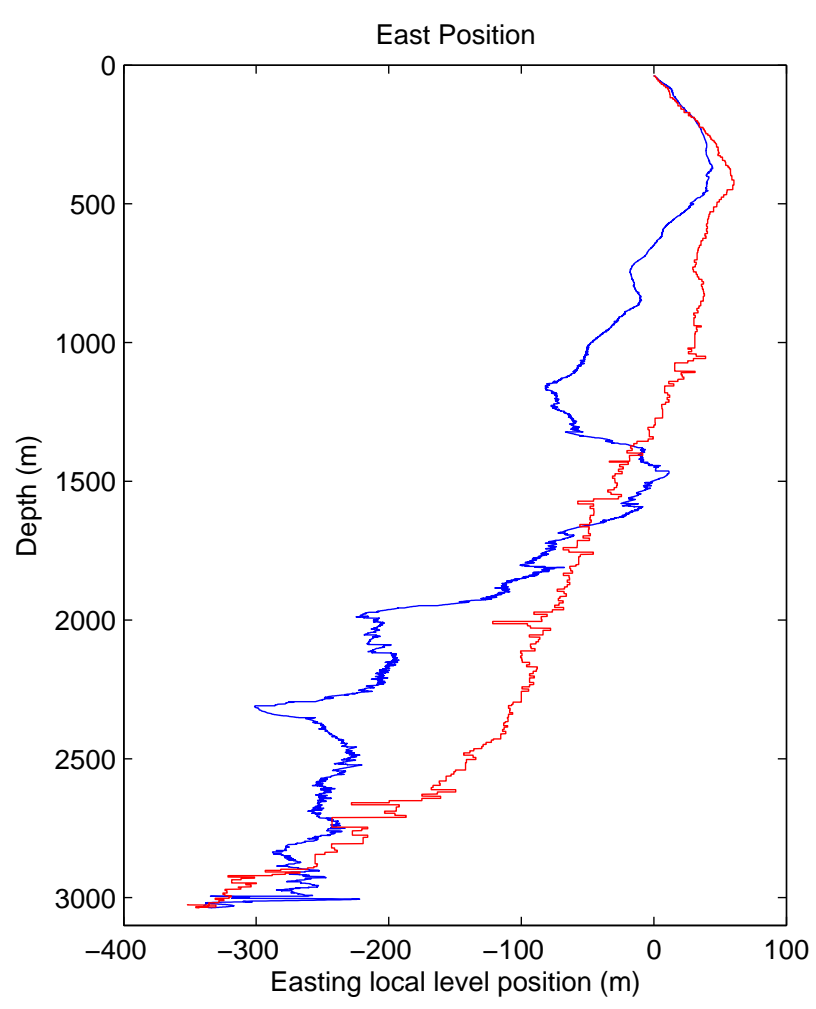

(b)

Fig. 15 - Sentry 220 (a) North and (b) East position estimates, comparing the filter output and the USBL output. Note the vertical scale is compressed compared to the exaggerated horizontal scale. 


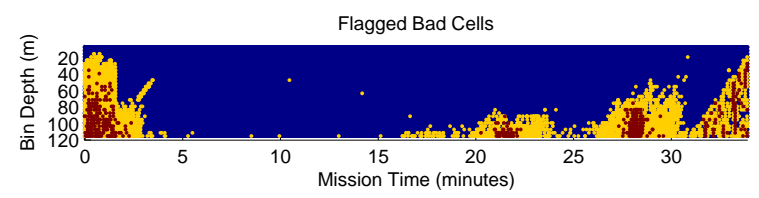

Fig. 16 - Data errors in the ADCP sensor for Sentry 273. The data in blue is good data prior to the $\chi^{2}$ test. The data in maroon has failed the percent good self-diagnostic of the sensor. The data in yellow exceeds the error velocity threshold.

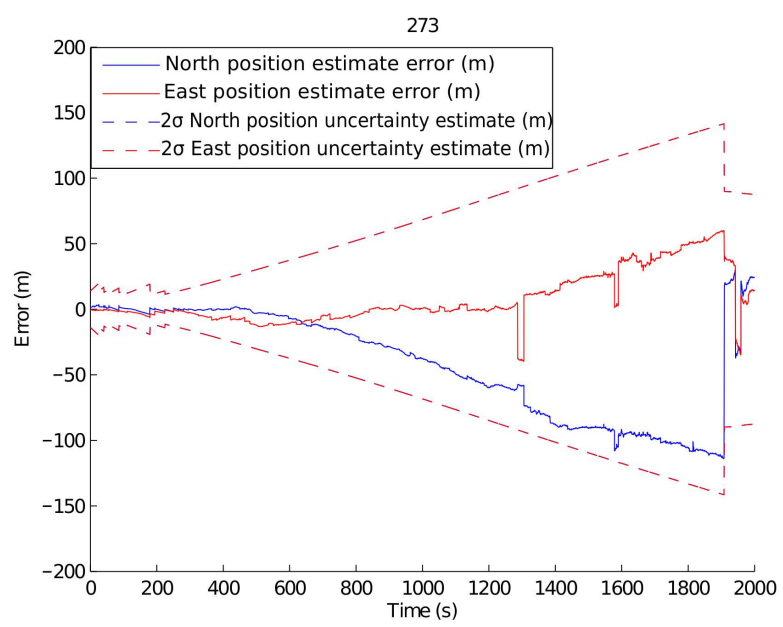

Fig. 17 - Sentry 273 position errors and uncertainty estimates for the filter. Filter is consistent as the errors are within the $2 \sigma$ bounds for the uncertainty estimates. The error is the difference between the estimate and the USBL ground truth. The unexpected jumps in error at approximately 1300, 1600 and 1950 seconds are due to USBL measurement errors.

show the data errors associated with the ADCP sensor during these missions, showing lower error rates than the Sentry 220 mission, although due to the reduced rate of $\mathrm{ADCP}$ measurements, the effective $\mathrm{ADCP}$ rate is lower. Sentry 273 has an effective ADCP rate of 1.6 $\mathrm{Hz}$, while Sentry 281 has an effective rate of $1.8 \mathrm{~Hz}$. In these missions, the ADCP runs on a timing trigger, and thus the effective rate of ADCP measurements is slightly less than Sentry 220 after including the effects of rejected measurements. Thus, difference in conditions between all the Sentry missions result in different effective ADCP rates.

Figure 17 plots the position error compared to the USBL and uncertainty estimates during the Sentry 273 descent. Unexpected jumps in error at approximately 1300, 1600 and 1950 seconds are due to USBL measurement errors - illustrating an additional redundancy benefit of the ADCP-aided method in the presence of

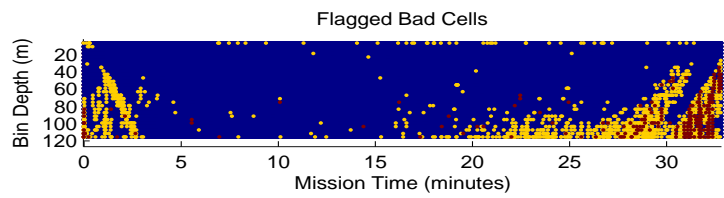

Fig. 18 - Data errors in the ADCP sensor for Sentry 281. The data in blue is good data prior to the $\chi^{2}$ test. The data in maroon has failed the percent good self-diagnostic of the sensor. The data in yellow exceeds the error velocity threshold.

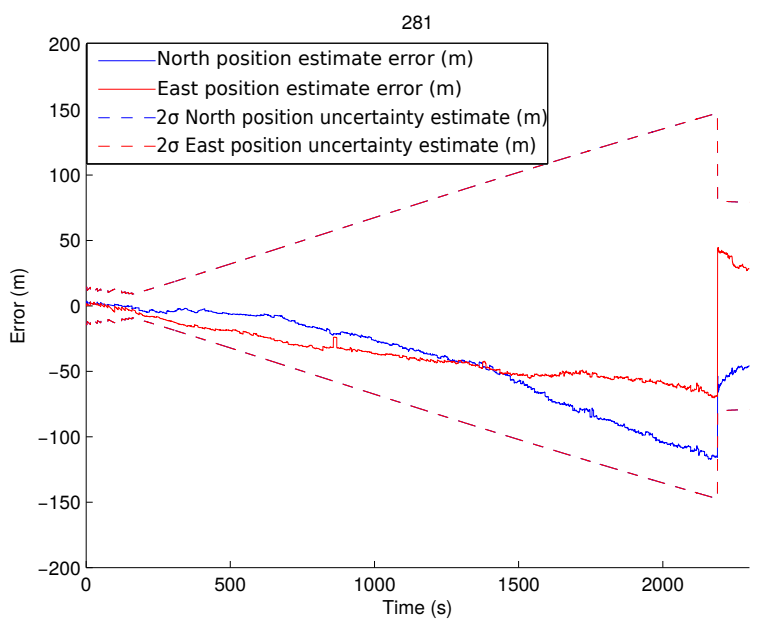

Fig. 19 - Sentry 281 position errors and uncertainty estimates for the filter. Filter is consistent as the errors are within the $2 \sigma$ bounds for the uncertainty estimates. The error is the difference between the estimate and the USBL ground truth.

noisy USBL. After $\sim 1700$ seconds, the estimation possesses $130 \mathrm{~m}$ of position uncertainty growth, with a 7.7 $\mathrm{cm} / \mathrm{s}$ position uncertainty growth rate prior to DVL bottom lock. Once DVL bottom lock is obtained, the position uncertainty is reduced to $74 \mathrm{~m}$ with a growth rate of $4.4 \mathrm{~cm} / \mathrm{s}$. This is comparable to Sentry 281 (Figure 19) which has a similar descent rate but higher effective ADCP rate due to less ADCP data errors. Sentry 281 has a position uncertainty growth rate of 6.8 $\mathrm{cm} / \mathrm{s}$ - lower than Sentry 273. This results in $138 \mathrm{~m}$ of position uncertainty growth over the $\sim 2000 \mathrm{~s}$ prior to DVL bottom lock. Once DVL bottom lock is obtained, the position uncertainty growth is reduced to $70 \mathrm{~m}$ with a rate of $3.5 \mathrm{~cm} / \mathrm{s}$. Thus the increased effective ADCP measurement rate has resulted in lower position uncertainty growth for these similar missions, illustrating the effect of increased information loss during the descent in a real-world scenario.

These results show that the ADCP-aided filter provides localization during long descents to deep ocean 
depths, despite detectable errors (beyond biases and noise) in the ADCP measurements. The method works in two different environments, further verifying the utility of this technique. Further work could include an IMU to detect errors and uncertainty reduction by incorporating more sensors and water current modeling into the estimation, along with spatial correlation modeling of the water current profile.

\section{Comparison of experimental results}

Table 4 compares the experimental results presented in this paper. Following DVL bottom lock, the simulated Sirius mission performs within $1 \%$ uncertainty per distance traveled, incorporating TDCP GPS for high accuracy initial velocity uncertainty, and ideal sampling conditions resulting in a $3 \mathrm{~Hz}$ effective ADCP measurement rate. Sirius experiments at the Nuggets and Freycinet sites show higher uncertainty in the initial velocity, reduced rate ADCP measurements and no IMU, resulting in higher information loss during the descent, reflected in the close to $20 \%$ uncertainty per distance traveled. Sentry 220 provides higher effective ADCP rates, during a longer mission, although the lack of IMU and information loss during the descent results in $12 \%$ uncertainty per distance traveled following DVL bottom lock. Given faster descent rates of $60 \mathrm{~cm} / \mathrm{s}$ for Sentry 273 and 281, the effects of information loss are reduced, resulting in $6 \%$ and $7 \%$ uncertainty per distance traveled respectively. Various drivers for the uncertainty per distance traveled exist for our method including initial velocity uncertainty, effective ADCP measurement rates, descent rate, and incorporation of an IMU.

\section{Conclusion}

This paper reports an ADCP-aided localization algorithm as an alternative form of georeferencing for vertical dives during AUV deployments and presents experimental results showing its performance over a variety of missions. It has shown the ability to achieve constrained error growth in position estimates by incorporating ADCP measurements into the navigation solution of a vehicle transiting between the sea surface and the seafloor. This is achieved by incorporating water current velocities as states in the estimation. Simulations show ideal circumstances and the potential performance of the ADCP-aided method. The method outlined also allows the incorporation of view-based SLAM techniques within the formulation, and an entirely autonomously localized mission was presented in previ- ous work. The method was also shown to operate on real world experimental data for multi-hour descents to depths up to $3 \mathrm{~km}$. Thus, the reported method can provide accurate AUV navigation for missions requiring accurate underwater navigation close to the seafloor but that lack DVL bottom-lock during descent and ascent.

These results have short and long term implications for improved AUV autonomy. In the short term, this method allows more flexible and cost-effective AUV operations by reducing the dependency on expensive infrastructure (e.g., tending vessels, LBL transponders). Even if these infrastructure are employed, this method provides redundancy against acoustic position measurement errors. Longer term benefits include new capabilities for extended duration missions and improved navigation in polar regions. Long-range AUVs (Hobson et al (2012); Furlong et al (2012)) are enabling the long-duration biogeochemical process studies necessary for understanding the effects of climate change on the ocean; however, many of these missions will occur in the mid-water column where our navigation capabilities are presently the weakest. The results herein provide a solution in the vertical degree of freedom and serve as a foundation for future work in horizontal ADCP-aided navigation (Medagoda et al (2015)). This method could also enable new polar AUV missions where the presence of ice precludes obtaining GPS measurements and there are increased demands on navigation and long duration autonomy (Crees et al (2010); Nicholls et al (2006)). These future applications pose new research challenges. For example, in the case of untended longterm monitoring and exploration using AUVs or underwater gliders, tighter constraints on power consumption are imposed which will limit the frequency of ADCP measurements (Todd et al (2011)). As shown in section 5, even infrequent ADCP measurements provide information which permits localization, and determining whether it is feasible for such platforms is an open problem. Additional future work will further validate this approach by obtaining real time raw IMU sensor acceleration and rotation rate data and verifying the potential performance of ADCP-aided navigation fused with IMU data.

\section{Acknowledgment}

This work is supported in part by NCRIS IMOS, the Australian Research Council (ARC), the New South Wales Government and the Woods Hole Oceanographic Institution. Sirius AUV data was obtained on cruises supported by the University of Tasmania and the IMOS AUV Facility program. We thank the cruise PIs (N. Barrett and C. Johnson), the officers and crew of the 


\begin{tabular}{|c|c|c|c|c|c|c|c|c|c|c|c|c|}
\hline \multirow{2}{*}{ Mission name } & \multirow{2}{*}{$F$} & \multirow{2}{*}{$D$} & \multirow{2}{*}{$T$} & \multirow{2}{*}{$D / T$} & \multicolumn{4}{|c|}{ Prior to DVL bottom lock } & \multicolumn{4}{|c|}{ After DVL bottom lock } \\
\hline & & & & & $E$ & $U$ & $U / T$ & $U / D$ & $E$ & $U$ & $U / T$ & $U / D$ \\
\hline Sirius simulation & $3 \mathrm{~Hz}$ & $720 \mathrm{~m}$ & $3600 \mathrm{~s}$ & $20 \mathrm{~cm} / \mathrm{s}$ & $12 \mathrm{~m}$ & $15 \mathrm{~m}$ & $0.4 \mathrm{~cm} / \mathrm{s}$ & 0.02 & $5 \mathrm{~m}$ & $7 \mathrm{~m}$ & $0.2 \mathrm{~cm} / \mathrm{s}$ & 0.01 \\
\hline Sirius Nuggets* & $1 \mathrm{~Hz}$ & $30 \mathrm{~m}$ & $150 \mathrm{~s}$ & $20 \mathrm{~cm} / \mathrm{s}$ & $27 \mathrm{~m}$ & $17 \mathrm{~m}$ & $11.3 \mathrm{~cm} / \mathrm{s}$ & 0.6 & $8 \mathrm{~m}$ & $6 \mathrm{~m}$ & $4 \mathrm{~cm} / \mathrm{s}$ & 0.20 \\
\hline Sirius Freycinet & $0.5 \mathrm{~Hz}$ & $62 \mathrm{~m}$ & $312 \mathrm{~s}$ & $20 \mathrm{~cm} / \mathrm{s}$ & $57 \mathrm{~m}$ & $27 \mathrm{~m}$ & $8.7 \mathrm{~cm} / \mathrm{s}$ & 0.43 & $10 \mathrm{~m}$ & $13 \mathrm{~m}$ & $4.2 \mathrm{~cm} / \mathrm{s}$ & 0.21 \\
\hline Sentry 220 & $1.9 \mathrm{~Hz}$ & $2141 \mathrm{~m}$ & $5354 \mathrm{~s}$ & $40 \mathrm{~cm} / \mathrm{s}$ & $251 \mathrm{~m}$ & $328 \mathrm{~m}$ & $6.1 \mathrm{~cm} / \mathrm{s}$ & 0.15 & $88 \mathrm{~m}$ & $254 \mathrm{~m}$ & $4.7 \mathrm{~cm} / \mathrm{s}$ & 0.12 \\
\hline Sentry 273 & $1.6 \mathrm{~Hz}$ & $1054 \mathrm{~m}$ & $1683 \mathrm{~s}$ & $61 \mathrm{~cm} / \mathrm{s}$ & $129 \mathrm{~m}$ & $130 \mathrm{~m}$ & $7.7 \mathrm{~cm} / \mathrm{s}$ & 0.13 & $15 \mathrm{~m}$ & $74 \mathrm{~m}$ & $4.4 \mathrm{~cm} / \mathrm{s}$ & 0.07 \\
\hline Sentry 281 & $1.8 \mathrm{~Hz}$ & $1189 \mathrm{~m}$ & $2026 \mathrm{~s}$ & $59 \mathrm{~cm} / \mathrm{s}$ & $135 \mathrm{~m}$ & $138 \mathrm{~m}$ & $6.8 \mathrm{~cm} / \mathrm{s}$ & 0.12 & $54 \mathrm{~m}$ & $70 \mathrm{~m}$ & $3.5 \mathrm{~cm} / \mathrm{s}$ & 0.06 \\
\hline
\end{tabular}

* Refer to Medagoda et al (2011)

$F=$ Effective ADCP measurement frequency (approximate, after error checking)

$D=$ Descent depth with ADCP only

$T=$ Descent time with ADCP only

$E=$ Position error (difference between USBL and ADCP-aiding)

$U=2 \sigma$ position uncertainty growth

Table 4 - A comparison of all results in this paper. Descent time with only ADCP includes attitude referencing from a compass or gyrocompass. Position error includes that added due to the USBL ground truth uncertainty. Position uncertainty excludes initial positioning uncertainty prior to exclusively ADCP-aided localization.

$R / V$ Challenger and the Sirius operations team (D. Mercer and G. Powell). Deep water data was obtained on cruises AT26-09 (PIs: G. Wheat, A. Fisher, and S. Hulme) and AT26-17 (PIs: J. Kinsey, T. Crone, and E. Mittelsteadt) through funding from National Science Foundation. We thank the officers and crew of the $R / V$ Atlantis and the Sentry operations team ( $\mathrm{Z}$. Berkowitz, A. Duester, J. Fujii, J. Hansen, M. Loebecker, S. Suman) for their assistance.

\section{References}

Atkinson C (2008) Analysis of shipboard ADCP data from RRS Discovery Cruise D324: RAPID Array Eastern Boundary. National Oceanography Centre Southampton, Technical Report

Brokloff N (1994) Matrix algorithm for Doppler sonar navigation. Brest, France, vol 2, pp 378-83

Brokloff N (1997) Dead reckoning with an ADCP and current extrapolation. In: OCEANS 1997. MTS/IEEE Conference Proceedings, vol 2, pp 994-1000

Brumley BH, Cabrera RG, Deines KL, Terray EA (1991) Performance of a broad-band acoustic Doppler current profiler. IEEE Journal of Oceanic Engineering 16(4):402-407

Camilli R, Reddy C, Yoerger D, Van Mooy B, Jakuba M, Kinsey J, McIntyre C, Sylva S, Maloney J (2010) Tracking hydrocarbon plume transport and biodegradation at Deepwater Horizon. Science 330(6001):201-204

Caress DW, Clague DA, Paduan JB, Martin JF, Dreyer BM, Chadwick WW Jr, Denny A, Kelley DS (2012) Repeat bathymetric surveys at 1-metre resolution of lava flows erupted at Axial Seamount in April 2011. Nature Geoscience 5(7):483-488, DOI $\{10.1038 /$ ngeo1496\}

Crees T, Kaminski C, Ferguson J, Laframboise J, Forrest A, Williams J, MacNeil E, Hopkin D, Pederson R (2010) UNCLOS under ice survey-A historic AUV deployment in the Canadian high Arctic. In: IEEE/MTS OCEANS, pp 1-8

Flenniken IV W (2005) Modeling inertial measurement units and analyzing the effect of their errors in navigation applications. Masters Thesis, Auburn University

Fossen T (1994) Guidance and control of ocean vehicles. John Wiley \& Sons, Ltd

Furlong ME, Paxton D, Stevenson P, Pebody M, McPhail SD, Perrett J (2012) Autosub long range: A long range deep diving AUV for ocean monitoring. In: Autonomous Underwater Vehicles (AUV), 2012 IEEE/OES, pp 1-7

German C, Yoerger D, Jakuba M, Shank T, Langmuir C, Nakamura K (2008) Hydrothermal exploration with the Autonomous Benthic Explorer. Deep-sea Research Part I-Oceanographic Research Papers 55(2):203-219, DOI 10.1016/j.dsr.2007.11.004

Gordon R (1996) Principles of Operation a Practical Primer. RD Instruments, San Diego

van Graas F, Soloviev A (2004) Precise velocity estimation using a stand-alone GPS receiver. Navigation(Washington, DC) 51(4):283-292

Healey A, Rock S, Cody S, Miles D, Brown J (1995) Toward an improved understanding of thruster dynamics for underwater vehicles. IEEE Journal of Oceanic Engineering 20(4):354-361

Hegrenaes O, Berglund E (2009) Doppler water-track aided inertial navigation for autonomous underwater vehicle. In: OCEANS 2009-EUROPE, 2009. OCEANS '09., pp 110, DOI 10.1109/OCEANSE.2009.5278307

Hegrenaes O, Hallingstad O (2011) Model-aided INS with sea current estimation for robust underwater navigation. IEEE Journal of Oceanic Engineering 36(2):316-337

Hobson BW, Bellingham JG, Kieft B, McEwen R, Godin M, Zhang Y (2012) Tethys-class long range AUVs - extending the endurance of propeller-driven cruising AUVs from days to weeks. In: 2012 IEEE/OES Autonomous Underwater Vehicles (AUV), pp 1-8

Hunt MM, Marquet WM, Moller DA, Peal KR, Smith WK, Spindell RC (1974) An acoustic navigation system. Technical Report WHOI-74-6, Woods Hole Oceanographic Institution, Woods Hole, Massachusetts 02543 USA

iXSea (Accessed 22-03-2012) PHINS brochure

Kaess M, Johannsson H, Roberts R, Ila V, Leonard J, Dellaert F (2011) isam2: Incremental smoothing and mapping with fluid relinearization and incremental variable reordering. In: 2011 IEEE International Conference on Robotics and Automation, IEEE, pp 3281-3288

Kelley DS, Karson JA, Früh-Green GL, Yoerger DR, Shank TM, Butterfield DA, Hayes JM, Schrenk MO, Olson EJ, Proskurowski G, et al (2005) A serpentinite-hosted ecosystem: the Lost City hydrothermal field. Science 307(5714):1428-1434

Kinsey JC, Whitcomb LL (2004) Preliminary field experience with the DVLNAV integrated navigation system for oceanographic submersibles. Control Engineering Practice 12(12):1541-1548, invited Paper 
Kinsey JC, Eustice RM, Whitcomb LL (2006) A survey of underwater vehicle navigation: Recent advances and new challenges. In: IFAC Conference of Manoeuvering and Control of Marine Craft

Kinsey JC, Yoerger DR, Jakuba MV, Camilli R, Fisher CR, German CR (2011) Assessing the Deepwater Horizon oil spill with the Sentry autonomous underwater vehicle. In: Intelligent Robots and Systems (IROS), 2011 IEEE/RSJ International Conference on, IEEE, pp 261-267

Kinsey JC, Yang Q, Howland JC (2014) Nonlinear dynamic model-based state estimators for underwater navigation of remotely operated vehicles. IEEE Transactions on Control Systems Technology (99):1-1

Lupton T (2010) Inertial slam with delayed initialisation. PhD thesis, University of Sydney

Lupton T, Sukkarieh S (2009) Efficient integration of inertial observations into visual SLAM without initialization. In: IEEE/RSJ International Conference on Intelligent Robots and Systems, pp 1547-1552, DOI 10.1109/ IROS.2009.5354267

Mahon I, Williams S, Pizarro O, Johnson-Roberson M (2008) Efficient View-Based SLAM Using Visual Loop Closures. IEEE Transactions on Robotics 24(5):1002 -1014, DOI 10.1109/TRO.2008.2004888

Martin S, Whitcomb L (2008) Preliminary results in experimental identification of 3-dof coupled dynamical plant for underwater vehicles. In: OCEANS 2008, IEEE, pp 1-9

McPhail SD, Pebody M (2009) Range-only positioning of a deep-diving autonomous underwater vehicle from a surface ship. IEEE Journal of Oceanic Engineering 34(4):669-677

Medagoda L, MV J, O P, SB W (2010) Water Column Current Profile Aided Localisation for Autonomous Underwater Vehicles. In: OCEANS 2010, IEEE, Sydney, Australia

Medagoda L, SB W, O P, MV J (2011) Water Column Current Profile Aided Localisation combined with Viewbased SLAM for Autonomous Underwater Vehicles. In: International Conference on Robotics and Automation 2011, IEEE, Shanghai, China

Medagoda L, Eilders M, Kinsey J (2015) Autonomous underwater vehicle navigation in a spatiotemporally varying water current field. In: IEEE International Conference on Robotics and Automation, pp 565 - 572

Napolitano F (2004) PHINS: THE AUTONOMOUS NAVIGATION SOLUTION. Sea Technology

Nicholls K, Abrahamsen E, Buck J, Dodd P, Goldblatt C, Griffiths G, Heywood K, Hughes N, Kaletzky A, LaneSerff G, McPhail S, Millard N, Oliver K, Perrett J, Price M, Pudsey C, Saw K, Stansfield K, Stott M, Wadhams P, Webb A, Wilkinson J (2006) Measurements beneath an Antarctic ice shelf using an autonomous underwater vehicle. Geophysical Research Letters 33(8), DOI 10.1029/2006GL025998

Paull L, Saeedi S, Seto M, Li H (2014) AUV Navigation and Localization: A Review. IEEE Journal of Oceanic Engineering

Peyronnet JP, Person R, Rybicki F (1998) POSIDONIA 6000 - a new long range highly accurate ultra short base line positioning system. Nice, Fr, vol 3, pp 1721-1727, DOI 10.1109/OCEANS.1998.726382

Schofield O, Ducklow HW, Martinson DG, Meredith MP, Moline MA, Fraser WR (2010) How do polar marine ecosystems respond to rapid climate change? Science 328(5985):1520-1523

Shih H, Payton C, Sprenke J, Mero T (2000) Towing basin speed calibration of acoustic Doppler current profiling in- struments. In: Joint Conference on Water Resources Engineering and Water Resources Planning and Management, American Society of Civil Engineers

Singh H, Armstrong R, Gilbes F, Eustice R, Roman C, Pizarro O, Torres J (2004a) Imaging coral i: Imaging coral habitats with the seabed auv. Subsurface Sensing Technologies and Applications 5(1):25-42

Singh H, Can A, Eustice R, Lerner S, McPhee N, Pizarro O, Roman C (2004b) Seabed AUV offers new platform for high-resolution imaging. EOS, Transactions of the AGU 85(31):289-294

Soon B, Scheding S, Lee H, Lee H, Durrant-Whyte H (2008) An approach to aid INS using time-differenced GPS carrier phase (TDCP) measurements. Gps Solutions 12(4):261-271

Stanway M (2011) Dead reckoning through the water column with an acoustic Doppler current profiler: Field experiences. In: OCEANS 2011, IEEE, pp 1-8

Stanway M (2012) Contributions to automated realtime underwater navigation. $\mathrm{PhD}$ thesis, Massachusetts Institute of Technology

Titterton D, Weston J (2004) Strapdown inertial navigation technology. Peter Peregrinus Ltd

Tivey MA, Johnson HP, Bradley AM, Yoerger DR (1998) Thickness of a submarine lava flow determined from nearbottom magnetic field mapping by autonomous underwater vehicle. Geophysical Research Letters 25(6):805-808

Todd RE, Rudnick DL, Mazloff MR, Davis RE, Cornuelle BD (2011) Poleward flows in the southern california current system: Glider observations and numerical simulation. Journal of Geophysical Research: Oceans (19782012) $116(\mathrm{C} 2)$

Visbeck M (2002) Deep velocity profiling using lowered acoustic Doppler current profilers: Bottom track and inverse solutions. Journal of Atmospheric and Oceanic Technology 19(5):794-807

Walter M, Eustice R, Leonard J (2007) Exactly sparse extended information filters for feature-based SLAM. The International Journal of Robotics Research 26(4):335-359

Whitcomb LL, Yoerger DR, Singh H, Howland J (1999) Combined Doppler/LBL based navigation of underwater vehicles. In: Proceedings of the the 11th International Symposium on Unmanned Untethered Submersible Technology, Durham, New Hampshire, USA

Williams S, Pizarro O, Mahon I, Johnson-Roberson M (2009) Simultaneous Localisation and Mapping and Dense Stereoscopic Seafloor Reconstruction Using an AUV. In: Experimental Robotics, Springer, pp 407-416

Williams S, Pizarro O, Webster J, Beaman R, Mahon I, Johnson-Roberson M, Bridge T (2010) Autonomous underwater vehicle-assisted surveying of drowned reefs on the shelf edge of the Great Barrier Reef, Australia. Journal of Field Robotics 27(5):675-697

Williams S, Pizarro O, Jakuba M, Johnson C, Barrett N, Babcock R, Kendrick G, Steinberg P, Heyward A, Doherty P, Mahon I, Johnson-Roberson M, Steinberg D, Friedman A (2012) Monitoring of benthic reference sites: Using an autonomous underwater vehicle. IEEE Robotics Automation Magazine 19(1):73-84, DOI 10.1109/MRA. 2011.2181772

Yoerger D, Jakuba M, Bradley A, Bingham B (2007) Techniques for deep sea near bottom survey using an autonomous underwater vehicle. International Journal of Robotics Research 26(1):41-54 